\title{
Evaluation of a Simple Model for the Acoustics of Bat Swarms
}

\author{
Mingyi Liu \\ Thesis submitted to the Faculty of the \\ Virginia Polytechnic Institute and State University \\ in partial fulfillment of the requirements for the degree of Master of Science \\ in \\ Mechanical Engineering \\ Rolf Müller \\ Nicole Abaid \\ Andrew Kurdila \\ September 15, 2016 \\ Blacksburg, Virginia
}

Keywords: Bat swarm, Jamming, Acoustics, Modeling, Simulation 
Evaluation of a Simple Model for the Acoustics of Bat Swarms

Mingyi Liu

(ABSTRACT) 
Bats using their biosonar while flying in dense swarms may face significant bioacoustic challenges, in particular mutual sonar jamming. While possible solutions to the jamming problem have been investigated multiple times in literature, the severity of this problem has received far less attention. To characterize the acoustics of bat swarms, a simple model of the acoustically relevant properties of a bat swarm has been set up and evaluated. The model contains only four parameters: bat spacial density, biosonar beamwidth, duty cycle, and a scalar measure for the smoothness of the flight trajectories. In addition, a threshold to define substantial jamming was set relative to the emission level. The simulations results show that all four model parameters can have a major impact on jamming probability. Depending on the combination of parameter values, situations with or without substantial jamming probabilities could be produced within reasonable ranges of all model parameters. Hence, the model suggests that not every bat swarm does necessarily experience grave jamming problem. A fitting process was introduced to describe the relationship between the four parameters and jamming probability, hence produced a function with jamming probability as output and four parameters as input. Since the model parameters should be comparatively easy to estimate for actual bat swarms, the simulation results could give researchers a way to assess the acoustic environment of actual bat swarms and determine cases where a study of biosonar jamming could be worthwhile.

Keywords: Bat swarm, Jamming, Acoustics, Modeling, Simulation 
Evaluation of a Simple Model for the Acoustics of Bat Swarms

Mingyi Liu

(GENERAL AUDIENCE ABSTRACT) 
Bats using their biosonar while flying in dense swarms may face significant bioacoustic challenges, in particular mutual sonar jamming. While possible solutions to the jamming problem have been investigated multiple times in literature, the severity of this problem has received far less attention. To characterize the acoustics of bat swarms, a simple model of the acoustically relevant properties of a bat swarm has been set up and evaluated. The model contains only four parameters: bat spacial density, biosonar beamwidth, duty cycle, and a scalar measure for the smoothness of the flight trajectories. In addition, a threshold to define substantial jamming was set relative to the emission level. The simulations results show that all four model parameters can have a major impact on jamming probability. Depending on the combination of parameter values, situations with or without substantial jamming probabilities could be produced within reasonable ranges of all model parameters. Hence, the model suggests that not every bat swarm does necessarily experience grave jamming problem. A fitting process was introduced to describe the relationship between the four parameters and jamming probability, hence produced a function with jamming probability as output and four parameters as input. Since the model parameters should be comparatively easy to estimate for actual bat swarms, the simulation results could give researchers a way to assess the acoustic environment of actual bat swarms and determine cases where a study of biosonar jamming could be worthwhile. 


\section{Dedication}

To my father, who always inspirs me. 


\section{Acknowledgments}

I could not finish this thesis without the help of Dr. Müller. Dr. Müller is always responsive with emails and is generous to give me suggestions even in detail figures. He always encourage me to present my work in GSA (Graduate Student) Assemble, BIST (Biology Inspired Science and Technology), and ASA. I learn a lot through those presentations and discussions. Dr. Müller works hard and is always enthusiastic about research. His expertise in biology, mathematics and computer science inspires me so much. I benefit a lot from the discussion he hosts every week.

I would thank Dr. Nicole Abaid for providing the bat's flying trajectory data. And special gratitude I own her for participating discussion in the early stage of this research and giving suggestions all through.

I would thank Dr. Tafti for participate in the early discussion and give me valuable suggestions.

I would also thank Dr. Hongxiao Zhu, for the solve of the mathematical equations, her expertise in Mathematics and statistics will always win my respect. 
I would thank Philip and Anupam for helping me setting up the super computers. Their help means a lot to me.

I would like to thank Dr. Rolf Müller, Dr. Nichole Abaid and Dr. Andrew Kurdila to serve as my committee member and guide me during my masters study in Virginia Tech.

First I can not help but express my highest solution to Dr. Müller, for his guidance showed in every step of this research. Dr. Müller works hard as well as being very patient. Again I am gratitude for the financial support Dr. Müller provided all the way.

I would thank Dr. Garvin, Dr. Moore, Dr. Taylor, who as committee member of BioBuild, for not only providing me the funding, but also for all the discussion related to bio-inspired technology. I was and will always remain an active member of BioBuild. What's more, your ideas and believes has rooted in my head deeply, I will pursuit bio-inspired technology in my entire life.

My gratitude also goes to Chinese Scholar Council (CSC) for providing me funding for those two years.

I own endless gratitude to my family, my mother, my grandfather, my brother and sisters, brothers and sisters in law. For their support and advice through all those two years. My special gratitude goes to my niece: Youyou and Tutu, my nephew Fang Hui, Liu Zhengxuan and Xixi. For the hope of future they show.

Even though he is not with us, my father has always been the source of inspiration. Every time I have difficulties or experience hard times, I will think what would he do if he 
faces such situation. I often image the world he lives in and the difficulty he faces, and then feel powerful myself.

My sincere thank goes to Anupam, Philip, Philex, Mingchen, Lvying, Uzair, Josef and all my other lab mates. It is their support provide me a very friendly environment that I enjoy for those three years.

I thank all the friends, family members, and teachers who inspires me. Their support are endless. I can not list here all here.

This thesis is dedicated to my wife, Yingying. For her unconditional love and support all the time. We are expecting much more in the future! 


\section{Contents}

1 Introduction $\quad 1$

1.1 Animal swarms and bat swarm .................... 1

1.2 Jamming avoidance strategy employed by bats . . . . . . . . . . . . . . . 2

1.3 Thesis organization . . . . . . . . . . . . . . . . . 4

2 Methods $\quad 6$

2.1 Bat swarm modeling . . . . . . . . . . . . . . . 6

2.2 Model parameters . . . . . . . . . . . . . . . . . . . 7

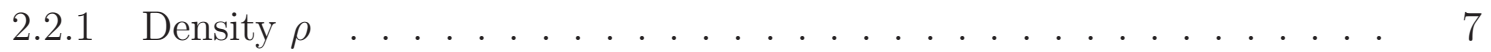

2.2.2 Duty cycle $\epsilon \ldots \ldots \ldots$. . . . . . . . . . . . . . 8

2.2.3 Beamwidth $\sigma \ldots \ldots \ldots \ldots \ldots$

2.2.4 Trajectory smoothness $\tau \ldots \ldots$. . . . . . . . . . . 12 
2.3 Periodic boundary simulation space . . . . . . . . . . . . . . . 15

2.4 Sum of acoustic input considering phase difference . . . . . . . . . . 16

2.5 Jamming threshold . . . . . . . . . . . . . . . . . . 17

3 Results $\quad 19$

3.1 Simulation setup . . . . . . . . . . . . . . . . . 19

3.2 Influence of parameters . . . . . . . . . . . . . . . . . 22

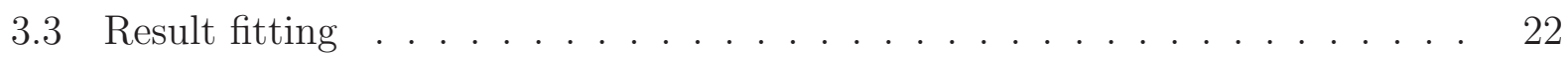

3.4 Overall result analysis . . . . . . . . . . . . . . . . . 25

4 Discussion $\quad 36$

4.1 Jamming contribution by individual parameters . . . . . . . . . . . 36

4.2 Jamming contribution by individual bat . . . . . . . . . . . . 37

4.3 Fitting process matching simulation results . . . . . . . . . . . . 38

4.4 Limitation by finite simulation space . . . . . . . . . . . . . . 39

4.5 Further steps to improve the simulation . . . . . . . . . . . . . . . 41

5 Future Work $\quad 42$

6 Conclusion $\quad 44$ 
6.1 A simple model for bat swarm . . . . . . . . . . . . . . . . . . . . 44

6.2 All four parameters are critical to jamming situation . . . . . . . . . 45

6.3 Not every bat swarm experience jamming . . . . . . . . . . . . . 45

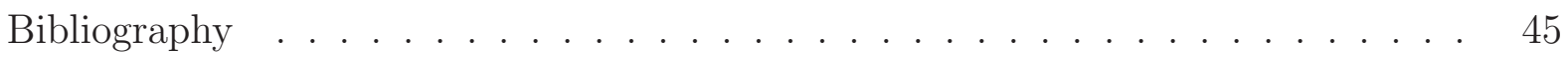

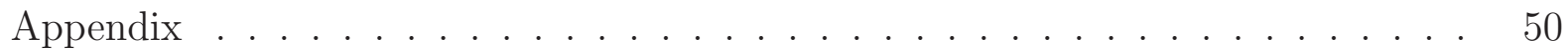

$\begin{array}{ll}\text { A Program Source } & 51\end{array}$ 


\section{List of Figures}

2.1 Model parameter bat spatial density $(\rho)$ with example realizations: a) $0.2 \mathrm{bats} / \mathrm{m}^{3}$, b) 1 bats $/ \mathrm{m}^{3}$, c) $2^{\text {bats }} / \mathrm{m}^{3}$. The bats fly in a volume of $10 \mathrm{~m} \times 10 \mathrm{~m} \times 10 \mathrm{~m}=1000 \mathrm{~m}^{3}$. $\quad 7$

2.2 Model parameter echolocation duty cycle $\epsilon$ with example realizations: a)20\%,

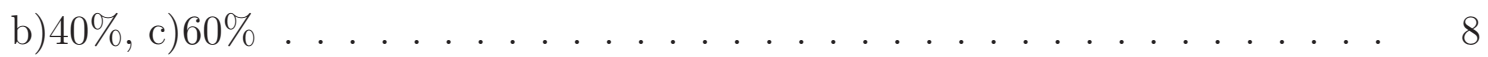

2.3 Model parameter beamwidth $(\sigma)$ : renderings of an example set of beam patterns with a) $10^{\circ}$, b) $30^{\circ}$, c) $90^{\circ}$ half-beamwidth at a gain level of $-3 \mathrm{~dB} . \quad . \quad 10$

2.4 Example beam pattern reconstructed from field measurement ${ }^{30} \ldots \ldots$

2.5 Example beam pattern from rotational-Gaussian model, with half-beamwidth

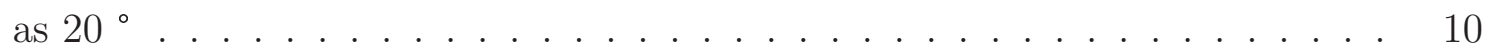


2.6 Model for the acoustic transmission channel between two bats ( bat $_{i}$ and bat $_{j}$ ). The bats have velocity vectors $\overrightarrow{v_{i}}$ and $\overrightarrow{v_{j}}$ respectively and are separated by distance $d$. The bearing angle of bat $j$ seen from bat $i$ is $\theta_{j i}$ and the bearing angle of bat $i$ seen from bat $j$ is $\theta_{i j}$. The respective channels gains are $g_{j i}$ and

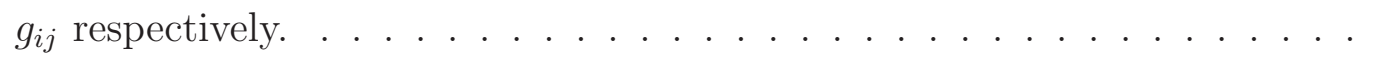

2.7 Model parameter flight trajectory smoothness (time constant $\tau$ ): Three example trajectory realizations are shown for each of the following time constant $(\tau)$ values: a) $0.01 \mathrm{~s}$, b) $0.1 \mathrm{~s}$, c) $0.3 \mathrm{~s} \ldots \ldots \ldots$

2.8 Fitting of field data (flight trajectory spectra) with a first-order low-pass trajectory model. Dashed line: average power spectrum of the trajectory captured in bat cave, solid line: first-order model fit. . . . . . . . . . . . . .

2.9 Examples of bat flight trajectories within the periodic boundary sampling space: Each of the five bats shown (in different gray shades) traverses the sampling volume several times (in this case, four times). . . . . . . . .

2.10 Example of jamming probabilities estimates: a) matrix of channel gains between all bats in the swarm simulation, b) input amplitude for each bat compared to the jamming threshold $(-20 \mathrm{~dB})$. Model parameter configuration: density $=0.03 \mathrm{bat} / \mathrm{m}^{3}$, time constant $\tau=0.2 \mathrm{~s}$, beamwidth $=30^{\circ}$, and duty cycle $=100 \%$.) . . . . . . . . . . . . . . . . 
3.1 Dependence of jamming probability on model parameters four example combinations of bat density $\rho$, duty cycle $\epsilon$, beamwidth $\sigma$, and trajectory time

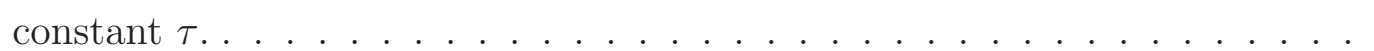

3.2 Illustration of saturation trend compared with sigmoid function (With all the rest parameter set to median values: $\tau=0.147 \mathrm{~s}$, duty cycle $=49.0 \%$, beamwidth $=49.2^{\circ}$, density $\left.=0.98 b a t / m^{3}\right) \ldots \ldots 23$

3.3 Influence of normalized parameter: density, $\tau$, beamwidth, and duty cycle .

3.4 Example jamming probability influenced by density and duty cycle . . . . .

3.5 Simulation result: jamming probability influenced by four parameters: duty cycle, density, beamwidth and time constant $\ldots \ldots \ldots \ldots 27$

3.6 Fitting result: fitting process to get numerical description of the result $\ldots 28$

3.7 Fitting error - with four parameters: duty cycle, density, beamwidth, and time constant $\tau \ldots \ldots \ldots \ldots \ldots \ldots \ldots \ldots \ldots \ldots \ldots \ldots \ldots \ldots \ldots \ldots$

3.8 Result shown by jamming v.s. beamwidth $\& \tau \ldots 30$

3.9 Simulation result v.s. four modeling parameters $\ldots \ldots \ldots 31$

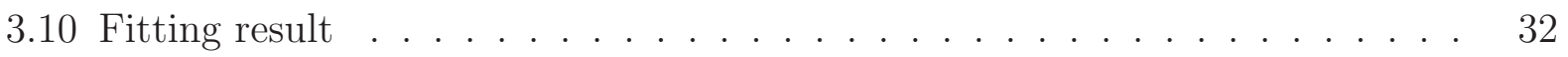

3.11 Absolute fitting error between simulation result and fitting result $\ldots . . .33$ 
3.12 Example distributions of jamming input amplitudes for bat that is not-jammed and jammed.(condition: time constant $\tau=0 \mathrm{~s}$, dutycycle $=100 \%$, beamwidth $=41^{\circ}$, density $=1$ bats $\left./ \mathrm{m}^{3}\right) \ldots \ldots \ldots \ldots$

3.13 Jamming probability as a function of the model's two density parameters (bat spacial density and duty cycle) a) minimum jamming probability achievable, b) maximum jamming probability achievable by varying the beamwidth and the trajectory smoothness time constant $(\tau)$ over the ranges of $\left[10^{\circ}, 60^{\circ}\right]$ for beamwidth and $[0 \mathrm{~s}, 0.3 \mathrm{~s}]$ for the trajectory time constant. . . . . . .

4.1 Influence of the simulation space on jamming probability, where duty cycle $=$ $100 \%$, and $\tau=0$ s . . . . . . . . . . . . . . . . . . 


\section{List of Tables}

3.1 simulation parameters . . . . . . . . . . . . . . . 21 


\section{Chapter 1}

\section{Introduction}

\subsection{Animal swarms and bat swarm}

Biological swarms are of broad scientific interest, because they are a model for how simple, local rules can be used to create complex patterns for large-scale coordination and collaboration ${ }^{1}$. In nature, swarms can be formed by animals as diverse as $a \operatorname{ats}^{2}$, fish ${ }^{3}$, birds ${ }^{4}$, and mammals ${ }^{5}$. Among the many animal groups that can form swarms, echolocating bats ${ }^{6}$ stand out because they employ an active sensory system, i.e., biosonar, to navigate, localize obstacle, and catch prey. The basic principle of (bio)sonar operation is to emit pulses, compare the returning echoes with pulses that triggered them, and interpret the differences (e.g., in arrival time, spectral composition) to estimate target properties. How such pulseecho comparisons can be conducted is most readily understood for a single (bio)sonar system 
operating in isolation. But for bats flying in dense swarms, matching and comparing echoes to pulses that triggered them could be a problem due to acoustic interference with pulses and echoes that are produced by other members of the swarm, i.e. the bat receives not only its own echo, but also emission and echoes from other bats, which serves as disturbance to that bat.

\subsection{Jamming avoidance strategy employed by bats}

Yet in nature, very rare occasions can we find that bats collide with each other, even when large aggregations of bats have to fly through a cluttered outlet of a cave. Through lab experiments and field recordings, various attempts have been made to explain how bats flying in swarm could avoid jamming with each other: shifting their emitted frequency bidirectionally away from the constant frequency jamming stimulus ${ }^{7} 89$, lower- and higher-frequency bats shifting their frequencies downwards and upwards to achieve less spectrum overlap when flying in a swarm ${ }^{10}$, shifting their peak frequency significantly to maximize differences between individuals ${ }^{11}$, flying in silence to avoid interference with sonar vocalizations of its neighbors ${ }^{12}$. Experiments found bats actually decrease their emission rate when there are a few neighbouring peers doing echolocation ${ }^{13}{ }^{14}$. But increase their emission rate, duration, and intensity when a lot of other bats are echolocating ${ }^{15} 16$, which might be explained as background noise to the bat. Jamming avoidance behavior not only happens conspecific but also between different species ${ }^{17}$. Researchers also found some bats use the echolocation call 
of its peers to locate feeding sites or even 'steal' food from the peers ${ }^{18}$, which instead of jamming each other, they actually take advantage of their peers. While at the same time, on board recordings found some species of bats in the wild actually did not use shifting spectrum to avoid jamming ${ }^{19}$.

However, a more fundamental question has received far little attention, namely how serious is the jamming problem bats in a swarm may face. How does jamming happen in a swarm? Which swarm property affects the the jamming situation? To address these questions, an analysis of a simple numerical model for the acoustics of a bat swarm is presented here. The model swarms are described by only four parameters: bat density, biosonar beamwidth, duty cycle, and a scalar measure for the smoothness of the flight trajectories. Results show that those four parameters does represent the bat swarm model well, and all those four parameters are significant to the bat model. Each of the four parameters is observable, i.e. could be measured in the field or in laboratory. With the results in this thesis,, our model could allow researchers working with bat swarms to determine the extent to how much bats may suffer from jamming in a given situation and identify scenarios where bats may need to rely on strategies to remedy jamming. Since there are only four modeling parameters, this model can be very easy for researchers to apply and get the jamming probability in a certain bat swarm.

As illustrated by hundreds of thousands of bats flying through a small opening of a cave, bat swarm is much more dense than any existing air vehicles flow. Yet, bats can deal with such a congestion smoothly. Investigating and finding out how bats did this can be a 
source of inspiration for the next generation of air traffic control system.

\subsection{Thesis organization}

In order to characterize the jamming situation in bat swarms, this thesis introduces a simple numerically construction of a bat swarm model, with only four parameters. Simulation is done based on the model and analysis the result obtained from simulation. The overall structure is organized as follow:

Chapter 1 introduces the background and the goal of this research. Literature review summarized state of art research on bat jamming and jamming avoidance strategies.

Chapter 2 describes the modeling in detail. The four parameters used to describe the bat swarm model: density, duty cycle, beamwidth, and trajectory smoothness was introduced and compared with real bats and bat swarm. Further effort was made to describe how the bats fly and interact with each other acoustically. The bats are confined in a $10 \times 10 \times$ $10 \mathrm{~m}^{3}$ space to simulate bats swarm flying in a large swarm. The sum of acoustic input considering phase effect and jamming threshold to tell a jammed and non-jamming bat was also introduced here.

Chapter 3 describes how the simulation is set up and implemented. The simulation results are presented in detail: how do the four parameters affect the jamming probability. A fitting process is introduced to describe the result numerically. Comparison is also made to find out the contribution of each individual parameter. Jamming contribution of one bat 
by other bats was also analyzed in detail.

Chapter 4 is the discussion part. This part is mainly about the analysis of the result and its implications. Analysis and conclusion based on the simulation is presented. This part also includes potential meaning and application of the result above. In addition, this part addresses how researcher can further improve the simulation to come even closer to the real bat swarm.

Chapter 5 is future work. In this chapter, the current air traffic jamming problem is highlighted and a brief introduction of current traffic management method is introduced. Comparison between the air traffic and bat swarm is made and how this research can be applied air traffic control management.

Chapter 6 is the conclusion part. A brief summary of what has been done in this thesis and the conclusion that can be made from this research. 


\section{Chapter 2}

\section{Methods}

\subsection{Bat swarm modeling}

In the simplified swarm model, each bat is represented by a random trajectory in threedimensional space and has no other interactions with other bats except acoustic interaction. The bats' sonar emissions consist of a regular sequences of pulses and pauses, each with a constant length in time. The directional characteristics of the bat biosonar are captured by a single beam pattern (relative gain as a function of direction) that describes emission and reception. Hence to specify this swarm model, a total of four parameters is needed, (i) the spatial density of the bat swarm, (ii) the duty cycle of the sonar call and (iii) the beamwidth of their sonar beam pattern, and - finally - (iv) the smoothness of their flight trajectories. The simplified interaction model is shown in Figure 2.6. 


\subsection{Model parameters}

\subsubsection{Density $\rho$}

The bats are assumed to be independently uniformly distributed in all three spatial dimensions. As a consequence, a single parameter, the density $\left(\rho\right.$, unit: bats $\left./ \mathrm{m}^{3}\right)$, is sufficient to specify the swarm distribution. For bat swarms exiting caves, densities as high as $0.63 \mathrm{bats} / \mathrm{m}^{3}$ have been reported, as an estimation derived from emergence rates, average flight speed, and cave cross-section ${ }^{21}$. Here, a range of density values from 0 bats $/ \mathrm{m}^{3}$ to 2 bats $/ \mathrm{m}^{3}$ has been examined in our simulation to cover all possible sparse as well as very dense regimes (Figure 2.1).
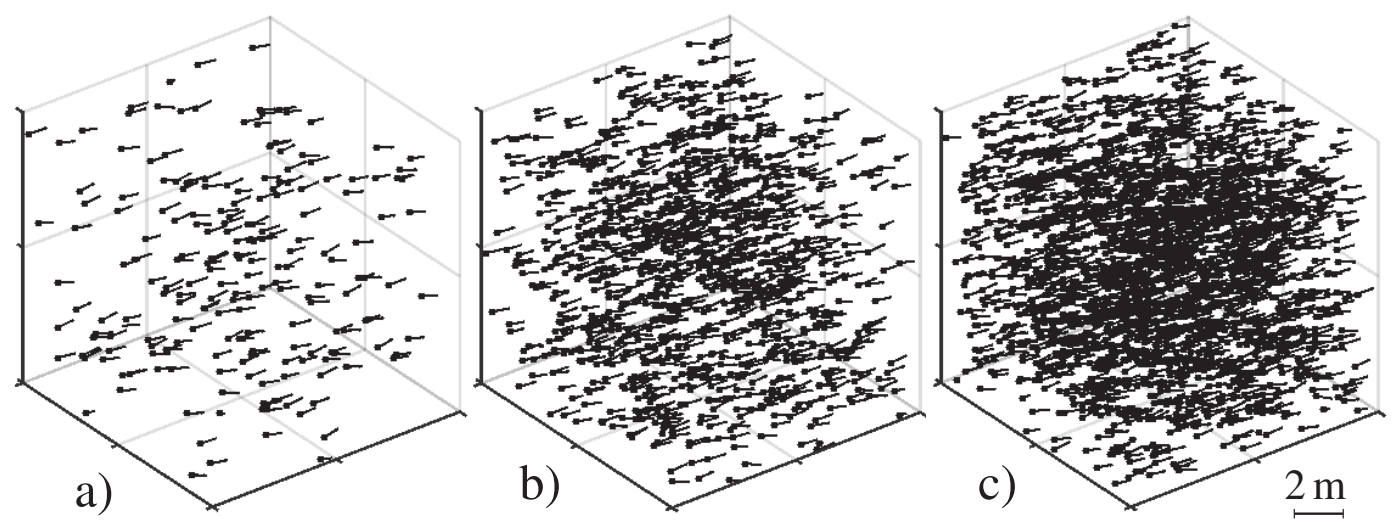

Figure 2.1: Model parameter bat spatial density $(\rho)$ with example realizations: a) $0.2 \mathrm{bats} / \mathrm{m}^{3}$, b) 1 bats $/ \mathrm{m}^{3}$, c) 2 bats $/ \mathrm{m}^{3}$. The bats fly in a volume of $10 \mathrm{~m} \times 10 \mathrm{~m} \times 10 \mathrm{~m}=1000 \mathrm{~m}^{3}$. 


\subsubsection{Duty cycle $\epsilon$}

The second parameter, temporal pulse density (duty cycle, $\epsilon$ ), describes the fraction of time (in percentage: $1 \%^{\prime}$ ) that is covered by sonar pulse emission as a percentage of the total duration of the pulse and one of the pauses that separate it from its neighbors. Together, the spatial density $\rho$ and the duty cycle $\epsilon$ describe the density of the bats' acoustic emission in space and time respectively. Duty cycles reported for bats range from $6.1 \%$ to $9.2 \%$ for low-duty cycle (LDC) bats and from $34.7 \%$ up to $56.3 \%$ for high-duty-cycle (HDC) bats $^{20}$. In nature, LDC bats form the largest swarms. The Mexican free-tailed bat (Tadarida brasiliensis), for example, forms the largest colonies and has a duty cycle of about $4.8 \%{ }^{20}$. Here, duty cycles ranging from 0 to $100 \%$ has been investigated to cover all possible values.

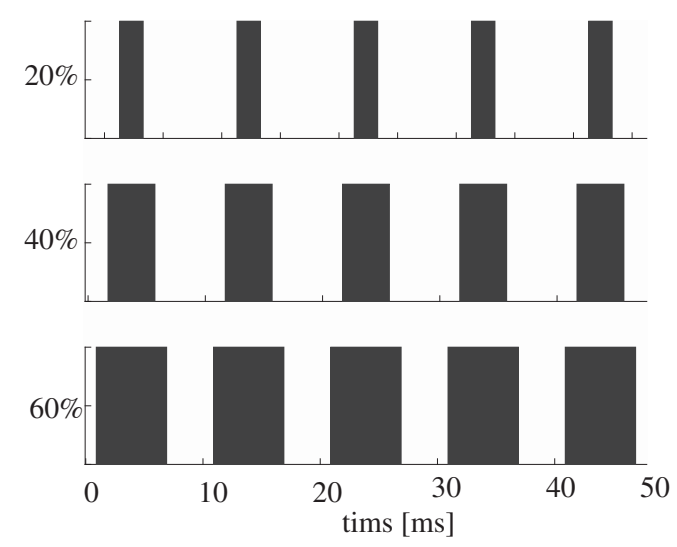

Figure 2.2: Model parameter echolocation duty cycle $\epsilon$ with example realizations: a)20\%, b) $40 \%$, c) $60 \%$ 


\subsubsection{Beamwidth $\sigma$}

Across different bat species, beam patterns differ in their shape as well as in overall beamwidth ${ }^{22}$.

Bats of the same species vary individually and even the same bat has been reported to change its beamwidth during individual behaviors ${ }^{232425}$. In the present model, all beam patterns were described by a rotation-symmetric Gaussian function that depended only on the angle between the direction for which the gain is to be determined and the heading of the bat, i.e., the maximum gain of the beam pattern is always aligned with the flight direction of the bat. This approach contained a further simplification in that the beam patterns were assumed to be independent of frequency. The emission and reception beam patterns were assumed to have an identical shape, beamwidth, and orientation. Under these simplifying assumptions, only a single parameter is needed to describe the distribution of emitted pulse amplitude and the receiver sensitivity with direction: the beamwidth. The beam pattern is described to be decay according to the shape of Gaussian distribution, can be quantified by the standard deviation $(\sigma)$ of the Gaussian distribution. Experiment and field study found beamwidth as low as $15^{\circ}$, and as high as $50^{\circ} 28$. Here, beamwidth (standard deviation, $\sigma$ ) values ranges from $10^{\circ}$ to $90^{\circ}$ were analyzed (Figure 2.3 ), to cover most of possible values

in bat's beam pattern. Comparison with reconstruction of bat's beam pattern in field ${ }^{30}$ and laboratory $^{31}$ indicates that the Gaussian shape fits with the recorded data well. 
a) beam width $=10^{\circ}$

b) beam width $=30^{\circ}$

c) beam width $=90^{\circ}$

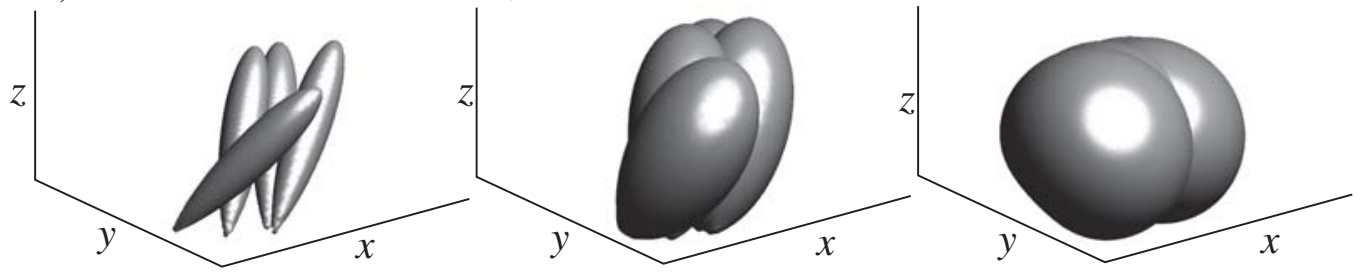

Figure 2.3: Model parameter beamwidth $(\sigma)$ : renderings of an example set of beam patterns with a) $10^{\circ}$, b) $30^{\circ}$, c) $90^{\circ}$ half-beamwidth at a gain level of $-3 \mathrm{~dB}$.
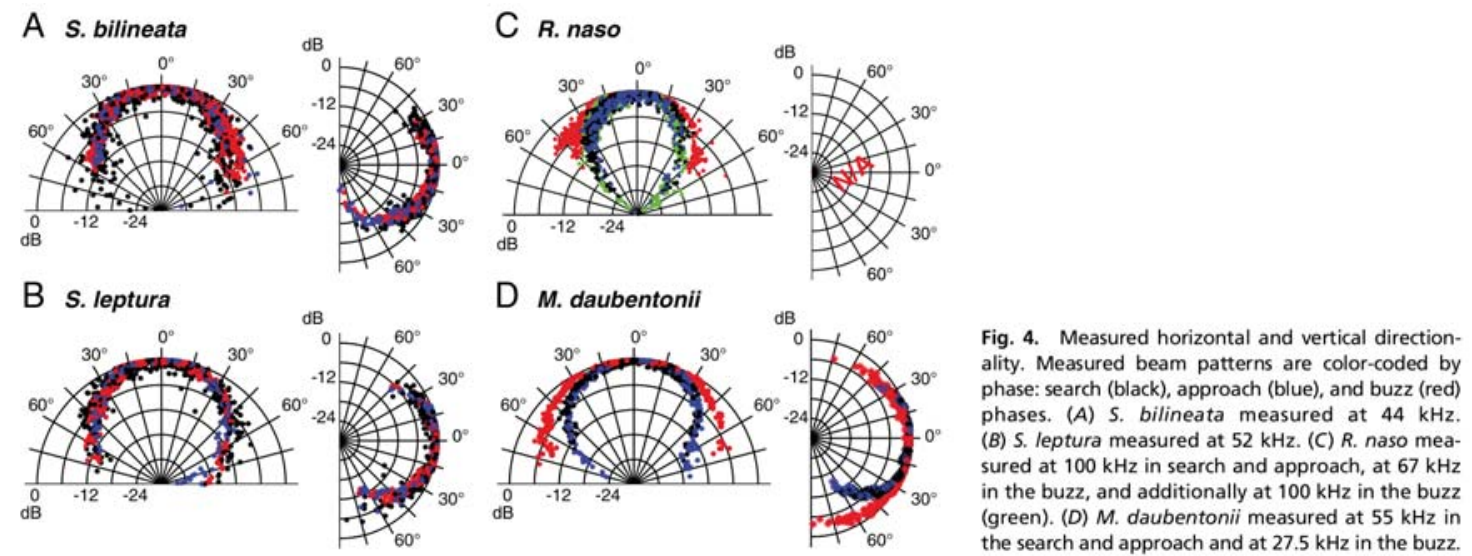

Figure 2.4: Example beam pattern reconstructed from field measurement ${ }^{30}$
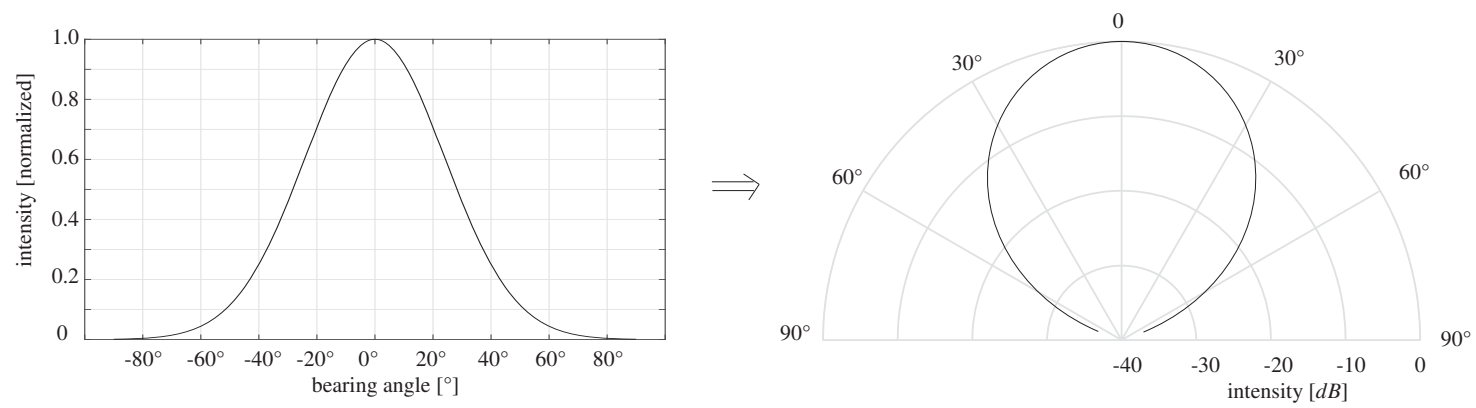

Figure 2.5: Example beam pattern from rotational-Gaussian model, with half-beamwidth as 
The modeling of the bat swarm is illustrated as following:

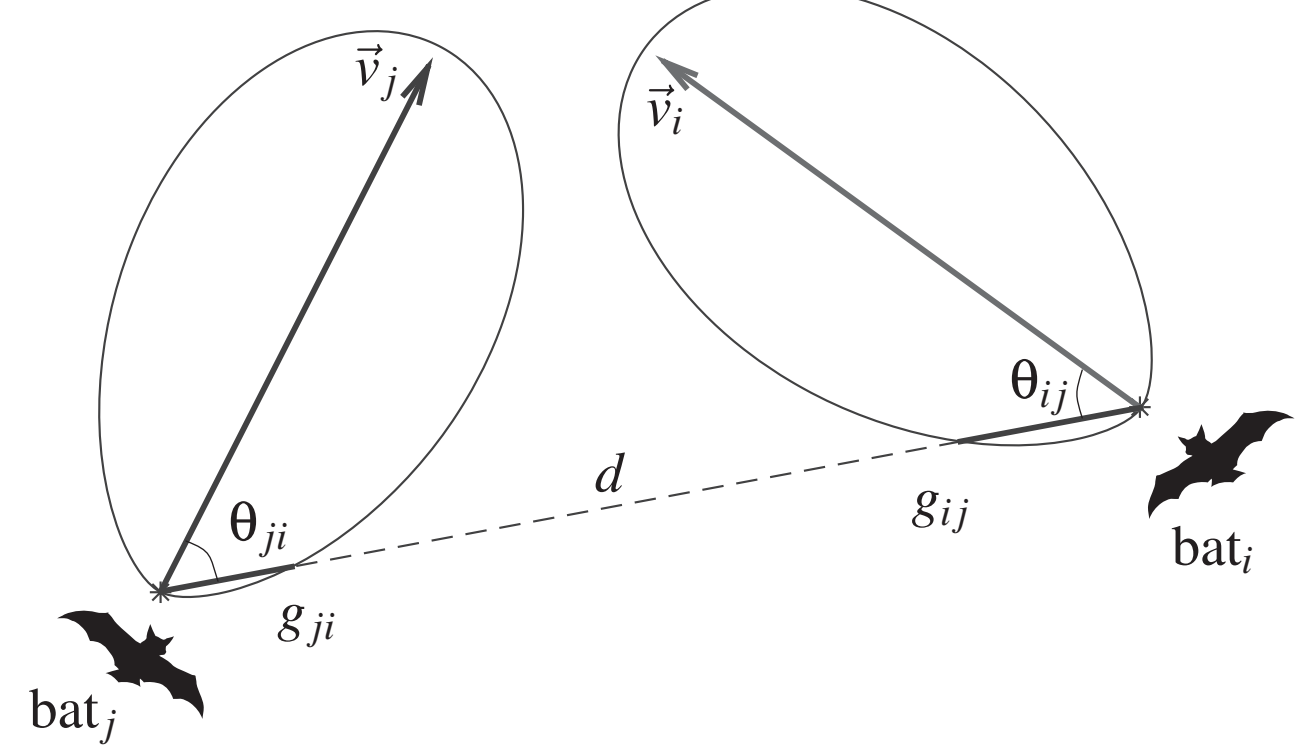

Figure 2.6: Model for the acoustic transmission channel between two bats (bat ${ }_{i}$ and bat $_{j}$ ). The bats have velocity vectors $\overrightarrow{v_{i}}$ and $\overrightarrow{v_{j}}$ respectively and are separated by distance $d$. The bearing angle of bat $j$ seen from bat $i$ is $\theta_{j i}$ and the bearing angle of bat $i$ seen from bat $j$ is $\theta_{i j}$. The respective channels gains are $g_{j i}$ and $g_{i j}$ respectively.

With this simplified beam pattern model, the input gain $g_{i j}$ from the $i^{\text {th }}$ (emitting) bat to $j^{\text {th }}$ (receiving) bat is:

$$
g_{i j}\left(\theta_{i j}, \theta_{j i}, d\right)= \begin{cases}\frac{0.2}{d} e^{\frac{-0.3466\left(\theta_{i j}^{2}+\theta_{j i}^{2}\right)}{\sigma^{2}}}, & d>0.2 \mathrm{~m} \\ e^{\frac{-0.3466\left(\theta_{i j}^{2}+\theta_{j i}^{2}\right)}{\sigma^{2}}}, & d \leq 0.2 \mathrm{~m}\end{cases}
$$

where $\theta_{i j}$ is the bearing angle of the $i^{\text {th }}$ bat seen from the $j^{\text {th }}$ bat, $\theta_{j i}$ the bearing angle of the $j^{\text {th }}$ bat seen from the $i^{\text {th }}$ bat, $d$ is the distance between the two bats, $\sigma$ is the half-beamwidth of the beam pattern. Spherical spreading losses were modeled by making amplitudes inversely 
proportional to distance $(d)$ for distances greater than a reference distance of $0.2 \mathrm{~m}$. Below this references, amplitudes were assumed to be constant at a maximum value that was normalized to 1 . This is set to avoid infinite gain when bats fly too close to each other. Constant -0.3466 is there to ensure that the emission (reception) power at equivalent to half beamwidth angle is half of that in right emission direction.

\subsubsection{Trajectory smoothness $\tau$}

The bats' flight trajectories were generated from a random input process with independent and identically distributed white Gaussian samples that were filtered with a first-order lowpass filters of adjustable bandwidth that is controlled via the time constant $(\tau)$. Trajectories with different degrees of smoothness were obtained by varying the time constant of the lowpass filter. Each random time sample was vector-valued with direction components in spatial dimensions $x, y$, and $z$. The values are taken to represent position. The differential equation for the filtered sequence of bat velocities is hence given by:

$$
\vec{p}_{n}=e^{-\frac{\delta t}{\tau}} \vec{p}_{n-1}+\left(1-e^{-\frac{\delta t}{\tau}}\right) \vec{w}_{n}
$$

where $\vec{p}_{n}=\left[p_{x}, p_{y}, p_{z}\right]$ is the position vector of the bat at the $n^{\text {th }}$ time step, $\vec{w}_{n}=\left[w_{x}, w_{y}, w_{z}\right]$ the sample from the Gaussian white-noise process that serves as input at the $n^{\text {th }}$ time step. $\delta t$ is the length of the time step and $\tau$ the time constant.

The discrete transfer function is:

$$
\frac{\vec{p}}{\vec{w}}=\frac{1-e^{-\frac{\delta t}{\tau}}}{1-e^{-\frac{\delta t}{\tau}} z}
$$


The transfer function magnitude of the first-order low-pass filter process (Equation 2.2) on a decibel scale is given by

$$
\begin{aligned}
H(\omega) & =\frac{1-e^{-\frac{\delta t}{\tau}}}{1-e^{-\frac{\delta t}{\tau}} e^{-j w \delta t}} \\
H(\omega) & =\frac{1-e^{-\frac{\delta t}{\tau}}}{1-\alpha \cos (w \delta t)+j e^{-\frac{\delta t}{\tau}} \sin (w \delta t)} \\
|H(\omega)| & =\frac{1-e^{-\frac{\delta t}{\tau}}}{\sqrt{\left[1-e^{-\frac{\delta t}{\tau}} \cos (w \delta t)\right]^{2}+\left[e^{-\frac{\delta t}{\tau}} \sin (w \delta t)\right]^{2}}} \\
|H(\omega)| & =\frac{1-e^{-\frac{\delta t}{\tau}}}{\sqrt{1+e^{-\frac{2 \delta t}{\tau}}-2 e^{-\frac{\delta t}{\tau}} \cos (w \delta t)}}
\end{aligned}
$$

where $\omega=2 \pi f$ is the angular velocity of input signal.

With different time constant $\tau$, different trajectory smoothness can be realized:

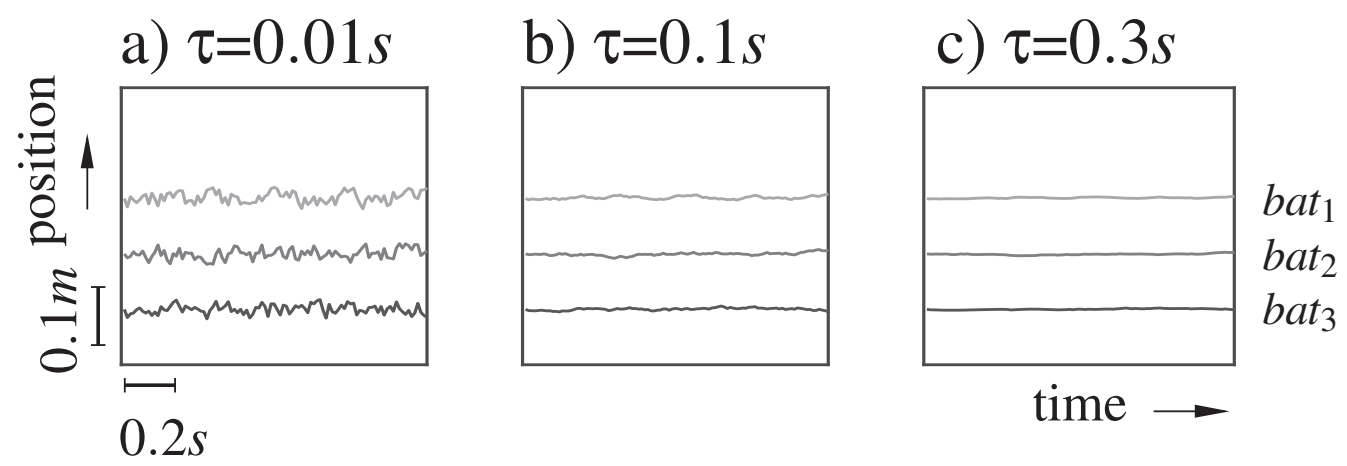

Figure 2.7: Model parameter flight trajectory smoothness (time constant $\tau$ ): Three example trajectory realizations are shown for each of the following time constant $(\tau)$ values: a) $0.01 \mathrm{~s}$, b) $0.1 \mathrm{~s}$, c) $0.3 \mathrm{~s}$.

From the flight trajectories shown above, we can find out that as $\tau$ value increases, the flying trajectory becomes more and more smooth. 
A set of 20 flight trajectories from (unidentified) bats exiting a cave situated on Mount Liantai, Shandong, China was obtained using a stereo-vision approach (GoPro Hero 3 cameras modified for infrared sensitivity). The transfer function magnitude (Equation 2.4) was fitted to power spectra obtained from the field flight trajectory data in a least-square sense. The time-constant value that yielded the best fit was found to be $\hat{\tau}=0.229 \mathrm{~s}$ (Figure 2.8 ).

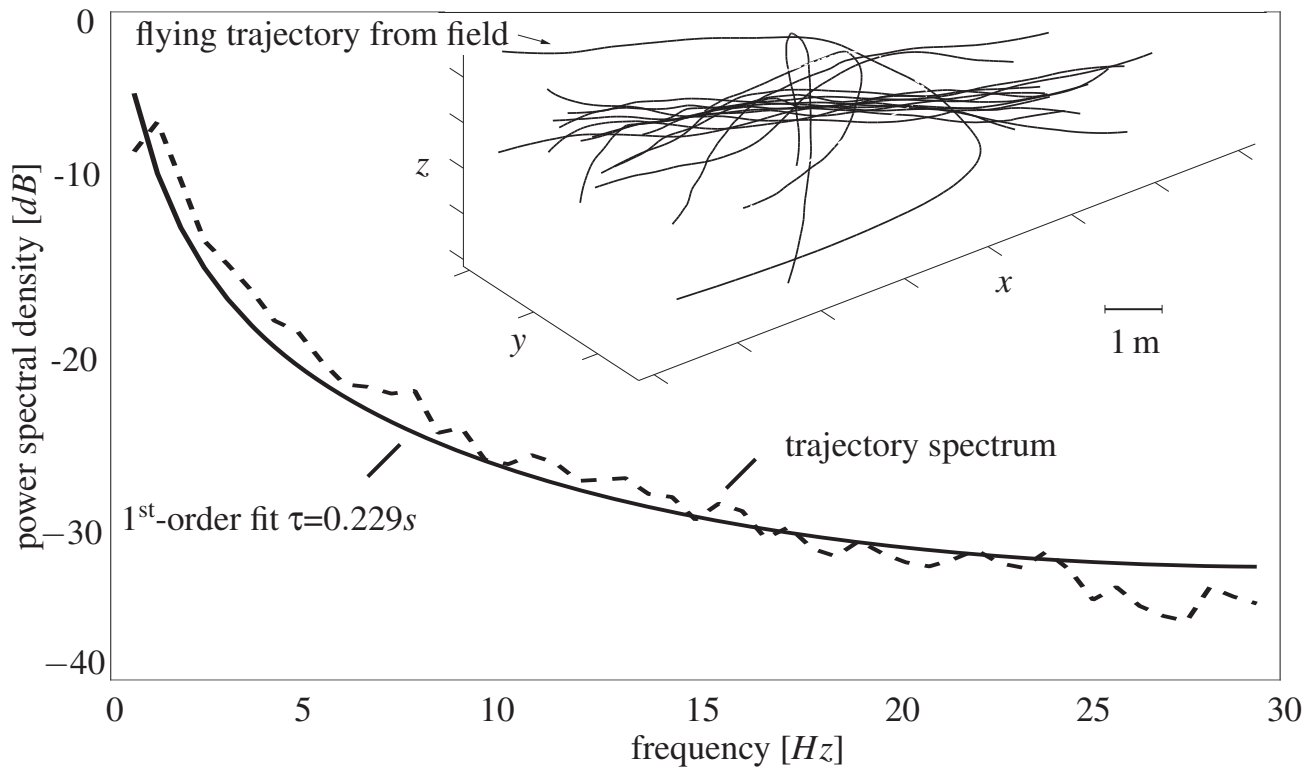

Figure 2.8: Fitting of field data (flight trajectory spectra) with a first-order low-pass trajectory model. Dashed line: average power spectrum of the trajectory captured in bat cave, solid line: first-order model fit.

In this model the range of time constant $\tau$ values analyzed was $0 \mathrm{~s}$ to $0.3 \mathrm{~s}$ to cover most trajectory smoothness. Flight speeds observed in field trip range up to $6.9 \mathrm{~m} / \mathrm{s}^{26}$, a reasonable value as $5 \mathrm{~m} / \mathrm{s}$ is set in the model. 


\subsection{Periodic boundary simulation space}

All bats' flight flight trajectories were initialized in parallel with one of the horizontal direction $(x)$ with speed of $5 \mathrm{~m} / \mathrm{s}$, i.e., the velocity components in the direction of the other two axes $(y$ and $z)$ was set to zero. The model was evaluated in a cubic spatial domain with an edge length of $10 \mathrm{~m} \times 10 \mathrm{~m} \times 10 \mathrm{~m}$ (hence the simulation space is $1000 \mathrm{~m}^{3}$ ). The initial position is uniformly distributed in the cubic space. This domain was set up to represent a segment within a large bat swarm where all swarm parameters remain constant. To achieve constant in the number of bats and their spatial distribution, periodic boundary conditions were used, i.e., bats that exited the cubic volume reappeared in the correspondent position on the opposite wall in the next time step, with the same velocity. The boundary space is illustrated as follow: 


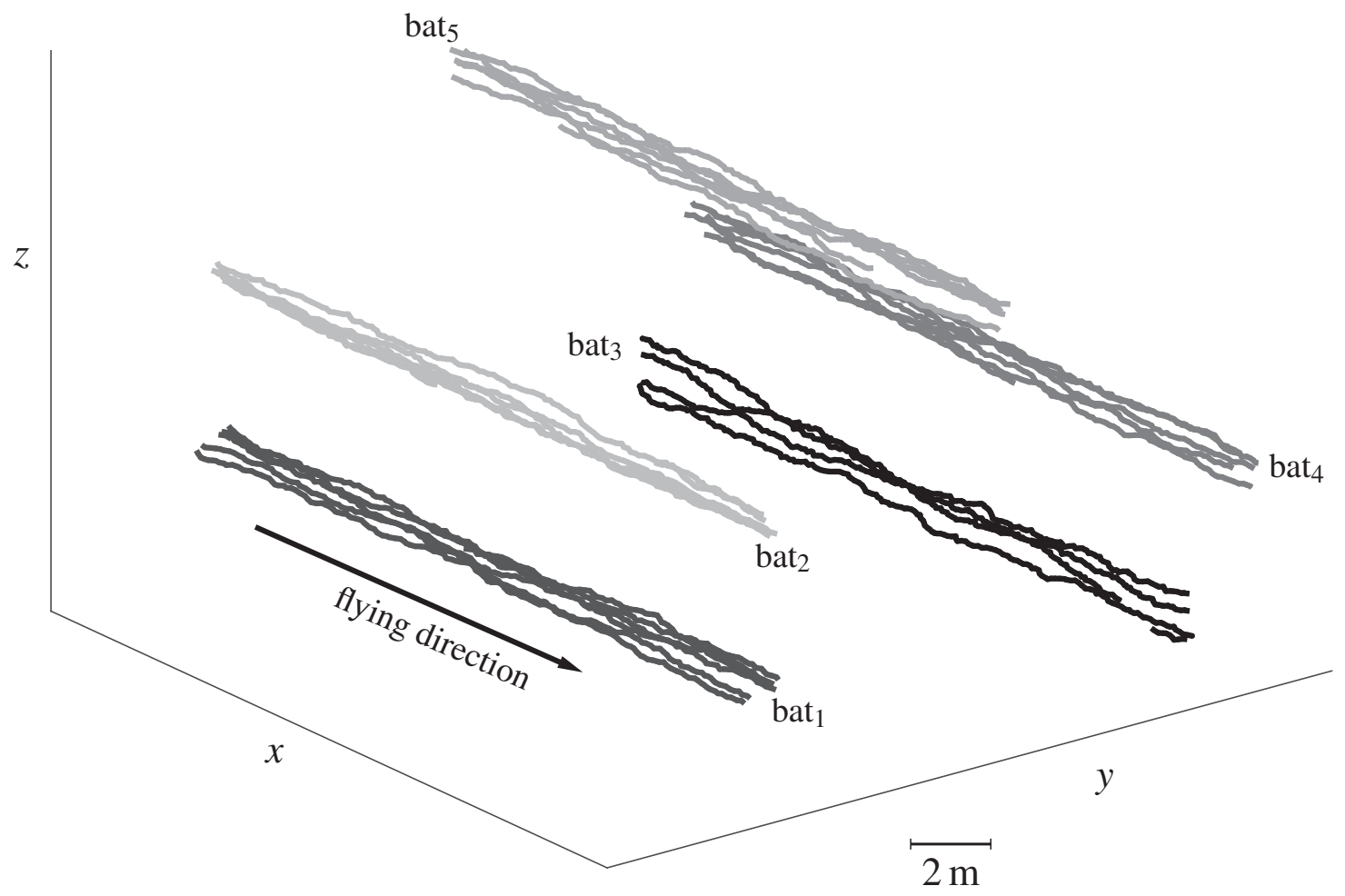

Figure 2.9: Examples of bat flight trajectories within the periodic boundary sampling space: Each of the five bats shown (in different gray shades) traverses the sampling volume several times (in this case, four times).

\subsection{Sum of acoustic input considering phase difference}

In order to compute the overall sonar input to individual bat in the swarm, the inputs to this bat from all other bats in the swarm were summed taking account the phase difference among the incoming signals: assuming all the acoustic emissions are of randomly distributed 
phase. The sum input from other bats to bat is

$$
\operatorname{Gain}_{i}=\sum_{j=1, \ldots, N, j \neq i} \operatorname{Gain}_{i j} \sin \left(w t+\phi_{i j}\right)
$$

where Gain $_{i j}$ and $\phi_{i j}$ are the amplitude and phase of $b a t_{j}$ 's emission, $w$ is the acoustic frequency of bat's emission and $t$ is time.

In the simulation code, the sum of two sinusoidal waves is implemented as follow:

$$
\begin{aligned}
& A \sin (\alpha)+B \sin (\beta) \\
= & A \sin (\alpha)+B \sin (\alpha+\beta-\alpha) \\
= & A \sin (\alpha)+B \sin (\alpha) \cos (\beta-\alpha)+B \cos (\alpha) \sin (\beta-\alpha) \\
= & {[A+B \cos (\beta-\alpha)] \sin (\alpha)+B \sin (\beta-\alpha) \cos (\alpha) } \\
= & \sqrt{[A+B \cos (\beta-\alpha)]^{2}+[B \sin (\beta-\alpha)]^{2}} \sin \left(\alpha+\operatorname{atan} \frac{B \sin (\beta-\alpha)}{A+B \cos (\beta-\alpha)}\right) \\
= & \sqrt{A^{2}+B^{2}+2 B \cos (\beta-\alpha)} \sin \left(\alpha+\operatorname{atan} \frac{B \sin (\beta-\alpha)}{A+B \cos (\beta-\alpha)}\right)
\end{aligned}
$$

\subsection{Jamming threshold}

A matrix of all interactions was computed (as shown in Figure 2.10). All inputs from other bats that is not muted by duty cycle, i.e. only $70 \%$ of the bats have input when duty cycle is $70 \%$ and the other $30 \%$ are muted, were summed up. A threshold value as $-20 \mathrm{~dB}$ (i.e. one tenth) of the sound pressure amplitude at $0.2 \mathrm{~m}$ right in front of the bat is $\mathrm{s}$ set to define jamming, those bats who get sum exceeds $-20 \mathrm{~dB}$ is defined as jammed bat and those less than $-20 \mathrm{~dB}$ is degined as not-jammed bat. Thus jamming probability is obtained by counting the portion of bats where input exceed jamming threshold. 


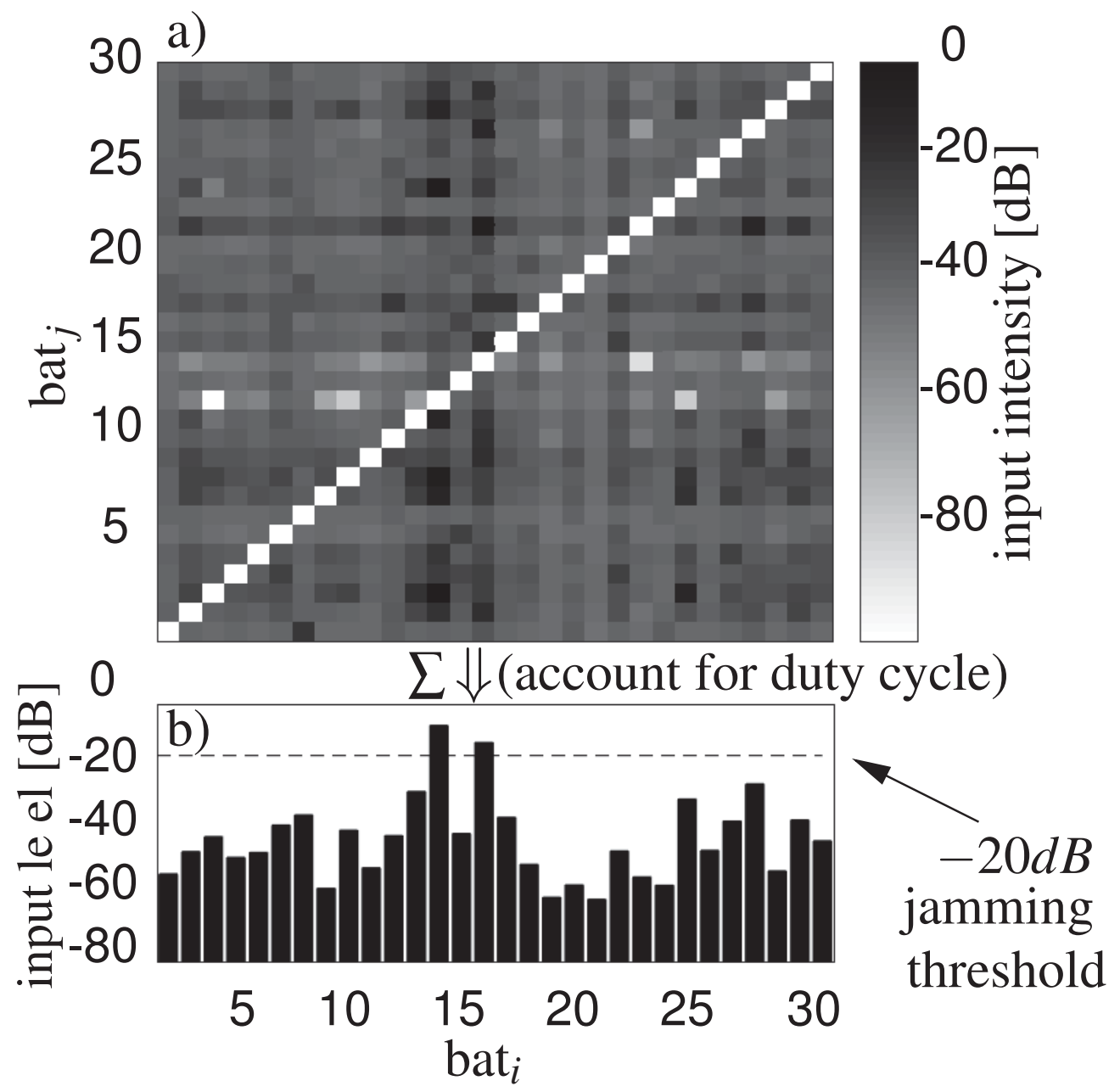

Figure 2.10: Example of jamming probabilities estimates: a) matrix of channel gains between all bats in the swarm simulation, b) input amplitude for each bat compared to the jamming threshold $(-20 \mathrm{~dB})$. Model parameter configuration: density $=0.03 \mathrm{bat} / \mathrm{m}^{3}$, time constant $\tau=$ $0.2 \mathrm{~s}$, beamwidth $=30^{\circ}$, and duty cycle $=100 \%$.) 


\section{Chapter 3}

\section{Results}

\subsection{Simulation setup}

For each simulation, all the bats were initially randomly distributed in $10 \mathrm{~m} \times 10 \mathrm{~m} \times 10 \mathrm{~m}$ three-dimensional space. As the simulation began, all the bats fly with speed $[5,0,0] \mathrm{m} / \mathrm{s}$. Acoustic interaction among them is obtained by calculating the acoustic gain between each of them. For each of the bat, acoustic input of all the rest of bats were summed together and compared with the jamming threshold: when it is over $-20 \mathrm{~dB}$, it is a jammed bat, otherwise, it is a not-jammed bat. Jamming probability is obtained by counting percentage of jammed bats: $\frac{\text { jammed bats }}{\text { total number of bats }} \times 100 \%$. Result were obtained by evaluating for $1.0 \mathrm{~s}$ and with a time step $\delta t 0.01 \mathrm{~s}$. Over all jamming probability is obtained by averaging all sets of results, to reduce the effect of noise generated by random input. 
Based on the model constructed, simulation runs by combining all different combinations of the four parameter, i.e. density $\rho:[0,2] \mathrm{bat} / \mathrm{m}^{3}$, time constant $\tau:[0,0.3] \mathrm{s}$, beamwidth $\sigma:\left[10^{\circ}, 90^{\circ}\right]$, duty cycle $\epsilon:[0 \%, 100 \%]$. For each of the parameter, 20 sampling points were evenly taken among parameter range, e.g. there are $20 \times 20 \times 20 \times 20=160000$ sets of model parameters and respective result for analysis. 
Table 3.1: simulation parameters

\begin{tabular}{|c|c|c|c|c|}
\hline parameter & $\operatorname{density} \rho\left[b a t / m^{3}\right]$ & duty cycle $\epsilon[\%]$ & beamwidth $\sigma\left[^{\circ}\right]$ & $\tau[\mathrm{s}]$ \\
\hline 1 & 0 & 0 & 10 & 0 \\
\hline 2 & 0.105 & 5.263 & 14.21 & 0.016 \\
\hline 3 & 0.211 & 10.53 & 18.42 & 0.032 \\
\hline 4 & 0.316 & 15.79 & 22.63 & 0.047 \\
\hline 5 & 0.421 & 21.05 & 26.84 & 0.063 \\
\hline 6 & 0.526 & 26.32 & 31.05 & 0.079 \\
\hline 7 & 0.632 & 31.58 & 35.26 & 0.095 \\
\hline 8 & 0.737 & 36.84 & 39.47 & 0.111 \\
\hline 9 & 0.842 & 42.11 & 43.68 & 0.126 \\
\hline 10 & 0.947 & 47.37 & 47.89 & 0.142 \\
\hline 11 & 1.053 & 52.63 & 52.11 & 0.158 \\
\hline 12 & 1.158 & 57.90 & 56.32 & 0.174 \\
\hline 13 & 1.263 & 63.16 & 60.53 & 0.190 \\
\hline 14 & 1.368 & 68.42 & 64.74 & 0.205 \\
\hline 15 & 1.474 & 73.68 & 68.95 & 0.221 \\
\hline 16 & 1.579 & 78.95 & 73.16 & 0.237 \\
\hline 17 & 1.684 & 84.21 & 77.37 & 0.253 \\
\hline 18 & 1.789 & 89.47 & 81.58 & 0.268 \\
\hline 19 & 1.895 & 94.74 & 85.79 & 0.284 \\
\hline 20 & 2.000 & 100 & 90 & 0.3 \\
\hline
\end{tabular}




\subsection{Influence of parameters}

Results show that all four parameters have major impact on the jamming probability:
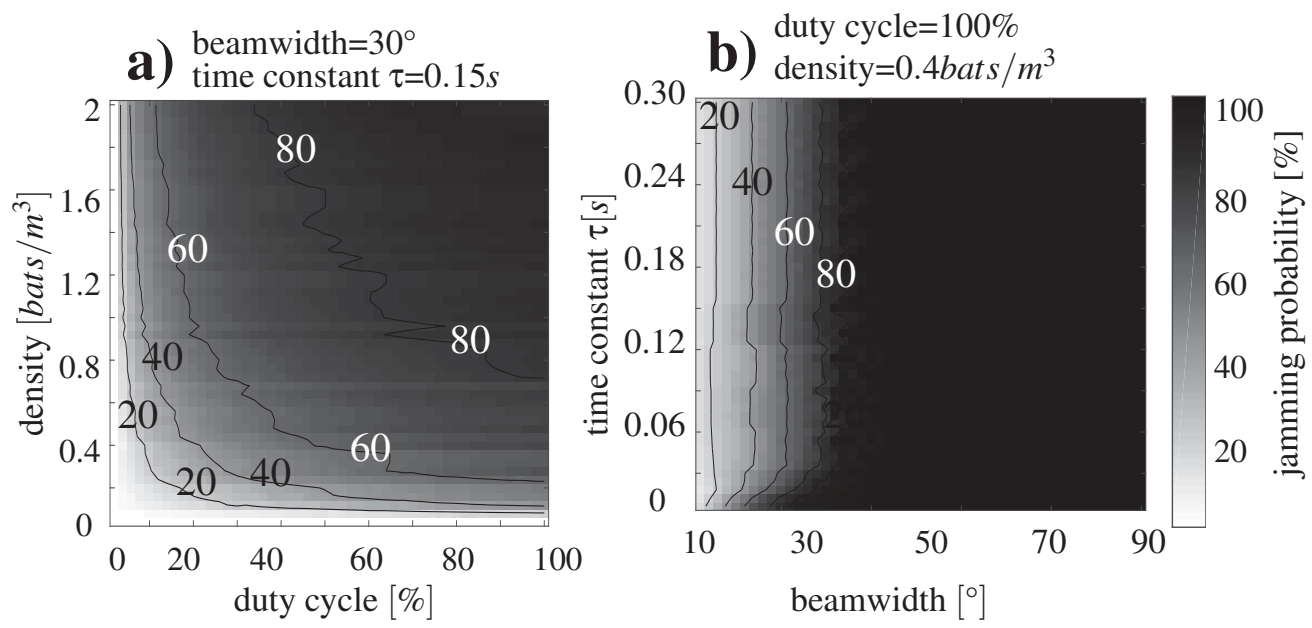

Figure 3.1: Dependence of jamming probability on model parameters four example combinations of bat density $\rho$, duty cycle $\epsilon$, beamwidth $\sigma$, and trajectory time constant $\tau$.

\subsection{Result fitting}

Jamming probabilities obtained in this way can be described by a model that is separable with respect to the four independent variables, i.e., the four model parameters: density $\rho$, duty cycle $\epsilon$, beamwidth $\sigma$, and time constant $\tau$. In order to get the numerical description of the results, a fitting process is introduced. For each individual parameter, the trend fits saturation function, which looks like the following: 


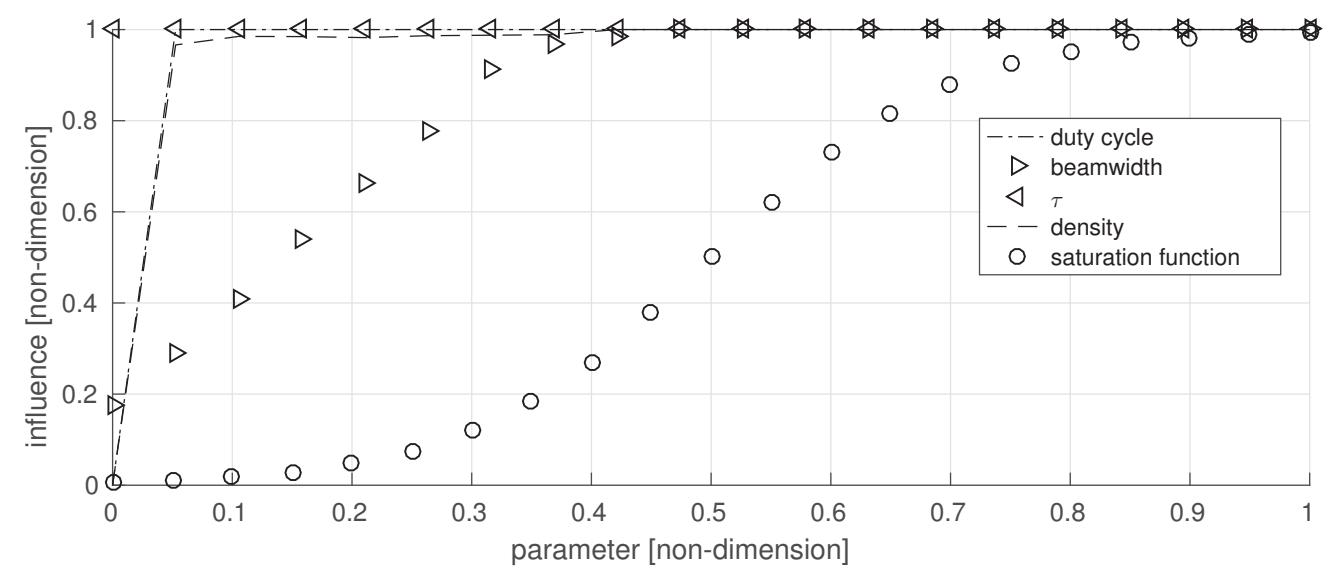

Figure 3.2: Illustration of saturation trend compared with sigmoid function (With all the rest parameter set to median values: $\tau=0.147 \mathrm{~s}$, duty cycle $=49.0 \%$, beamwidth $=49.2^{\circ}$, density $\left.=0.98 b a t / m^{3}\right)$

The sigmoid function is described as:

$$
p=\frac{a}{1+e^{b(\text { parameter }-c)}}
$$

where $b=[a, b, c]$ are parameters for the fitting model.

All the four parameters are assumed independent and the over all function described as:

$$
p=\frac{a}{\left(1+e^{b_{1}\left(\rho-c_{1}\right)}\right)\left(1+e^{b_{2}\left(\tau-c_{2}\right)}\right)\left(1+e^{b_{4}\left(\sigma-c_{4}\right)}\right)\left(1+e^{b_{3}\left(\epsilon-c_{3}\right)}\right)}
$$

where $\rho$ is the density of the bat swarm, time constant $\tau$ is the length of the first order filter, $\epsilon$ is the duty cycle of the bats, $\sigma$ is the beamwidth of the beam pattern, and the estimation matrix is $\left[a, b_{1}, b_{2}, b_{3}, b_{4}, c_{1}, c_{2}, c_{3}, c_{4}\right]$. After non-linear least square fit from MatLAB (fitnlm function), get fitting result as $\vec{b}=[101.5 ;-87.25 ;-5097 ;-0.1325 ;-1.821 ; 0.08058 ;-$ 
0.441;21.86;4.025]. So the jamming probability's relationship with the four parameter is described as:

$$
p=\frac{101.5}{\left(1+e^{-87.25(\rho-0.0805)}\right)\left(1+e^{-5097(\tau+0.441)}\right)\left(1+e^{-0.1325(\sigma-21.86)}\right)\left(1+e^{-1.821(\epsilon-4.025)}\right)}
$$

In order to compare the importance of the four simulation parameters to jamming probability, we normalize all four parameters to range $[0,1]$ and redo the fitting, and get fitting result as follow: $b=[101.5 ;-174.5 ;-1529 ;-10.60 ;-182.1 ; 0.04029 ;-1.470 ; 0.1483 ; 0.04025]$, which is plot as follow:

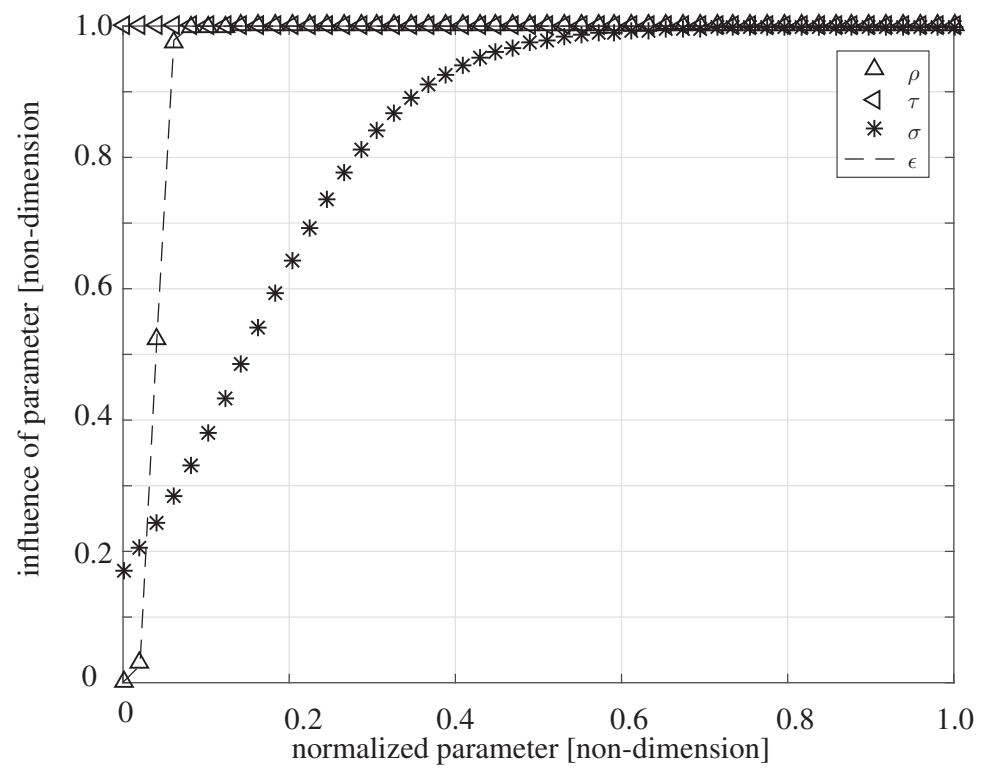

Figure 3.3: Influence of normalized parameter: density, $\tau$, beamwidth, and duty cycle

From the figure we can see that density and duty cycle have very strong influence on the final result and reach saturation early: saturat at less than $10 \%$ of the parameter range. Beamwidth also has very strong effect and reach saturation through $[0,40] \%$ of its 
parameter range. Because $\tau$ only has influence in a limited region ( $\tau$ only has effect for $[10,40]^{\circ}$ beamwidth), the fitting process does not capture the influence. This issue will be addressed in the discussion part.

\subsection{Overall result analysis}

In this section, we will look into the final overall result (final result is $20 \times 20 \times 20 \times 20$ dot simulation space, including both simulation and fitting result). First, we introduce the jamming probability v.s. duty cycle and density figure:

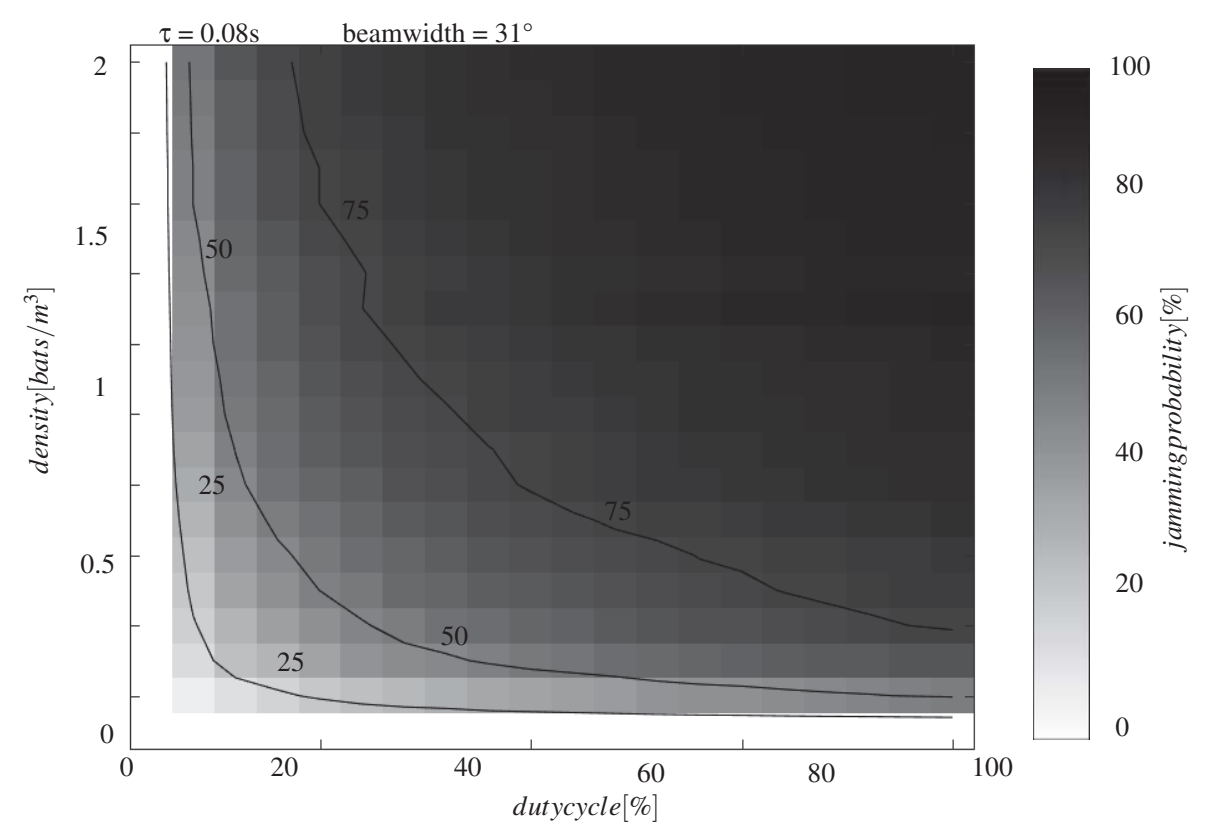

Figure 3.4: Example jamming probability influenced by density and duty cycle

From Figure 3.4 we can see the positive correlation between jamming probability 
and duty cycle \& density (which is similar to 3.1). Then we add another two parameters: beamwidth and $\tau$, we get the overall simulation result: 


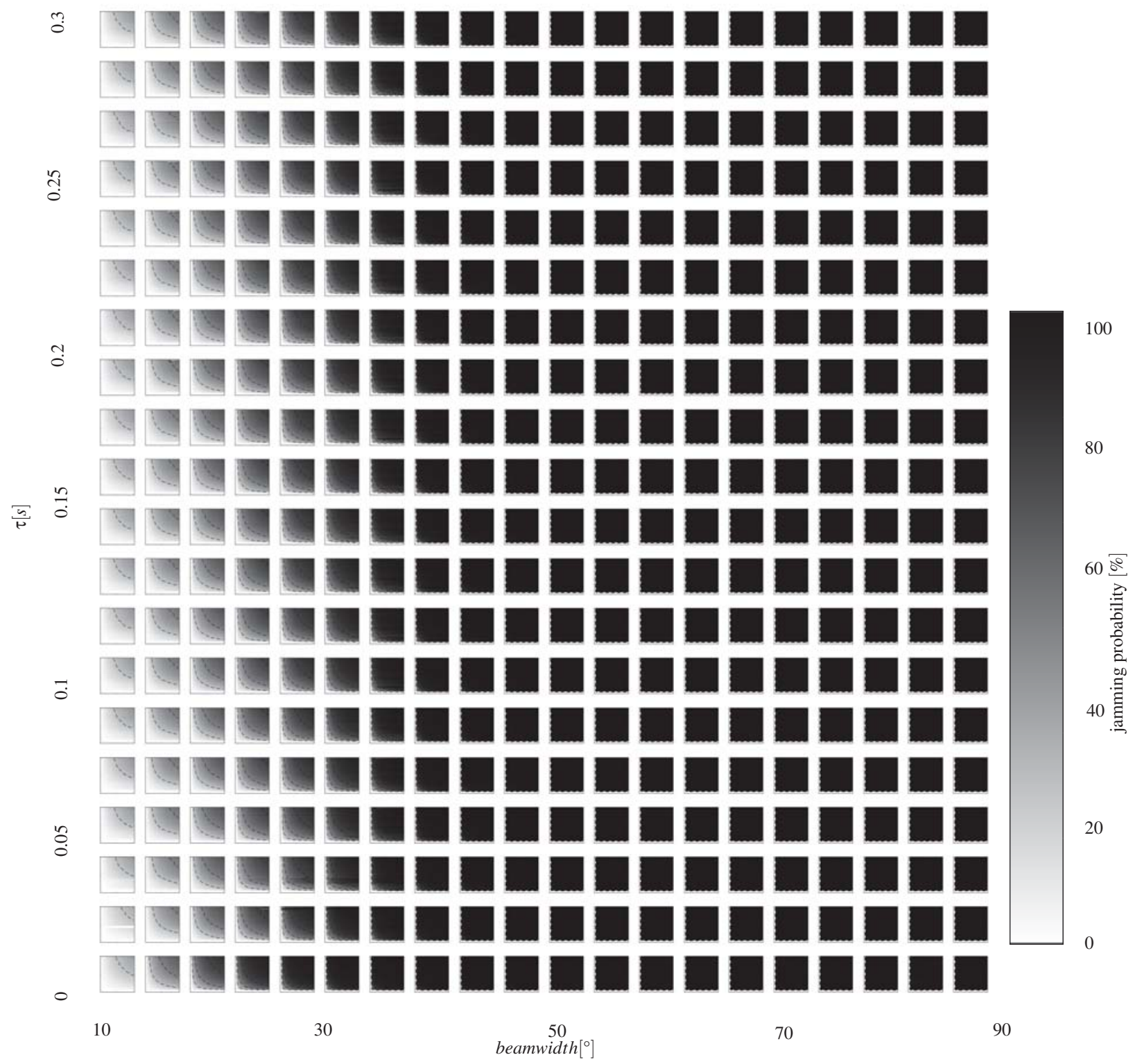

Figure 3.5: Simulation result: jamming probability influenced by four parameters: duty cycle, density, beamwidth and time constant $\tau$ 
With the fitting estimation obtained in the "Result fitting" section, we can plot the overall fitting result:

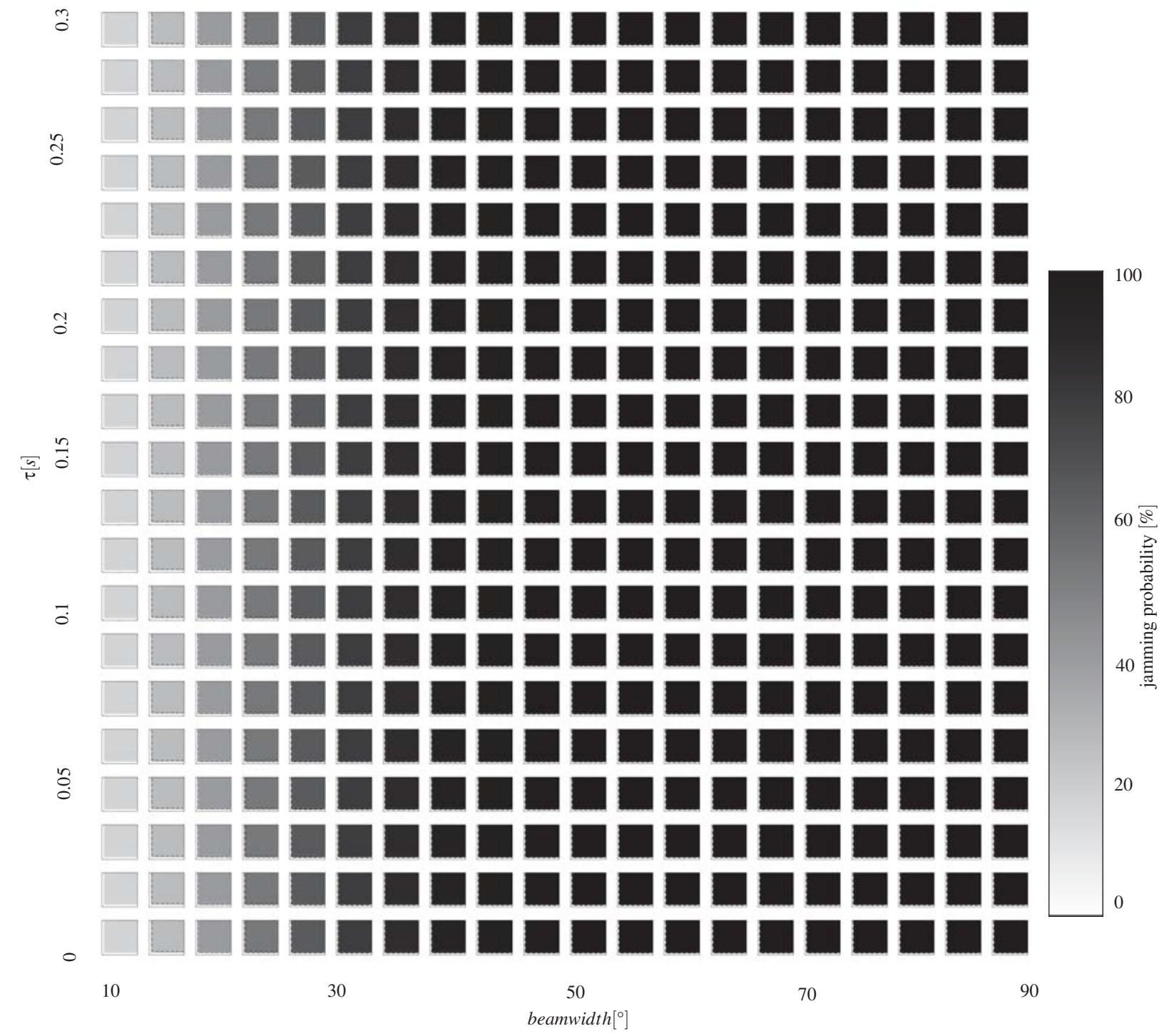

Figure 3.6: Fitting result: fitting process to get numerical description of the result 
fitting $\left._{\text {result }} \mid\right)$ can be obtained and shown as follow:

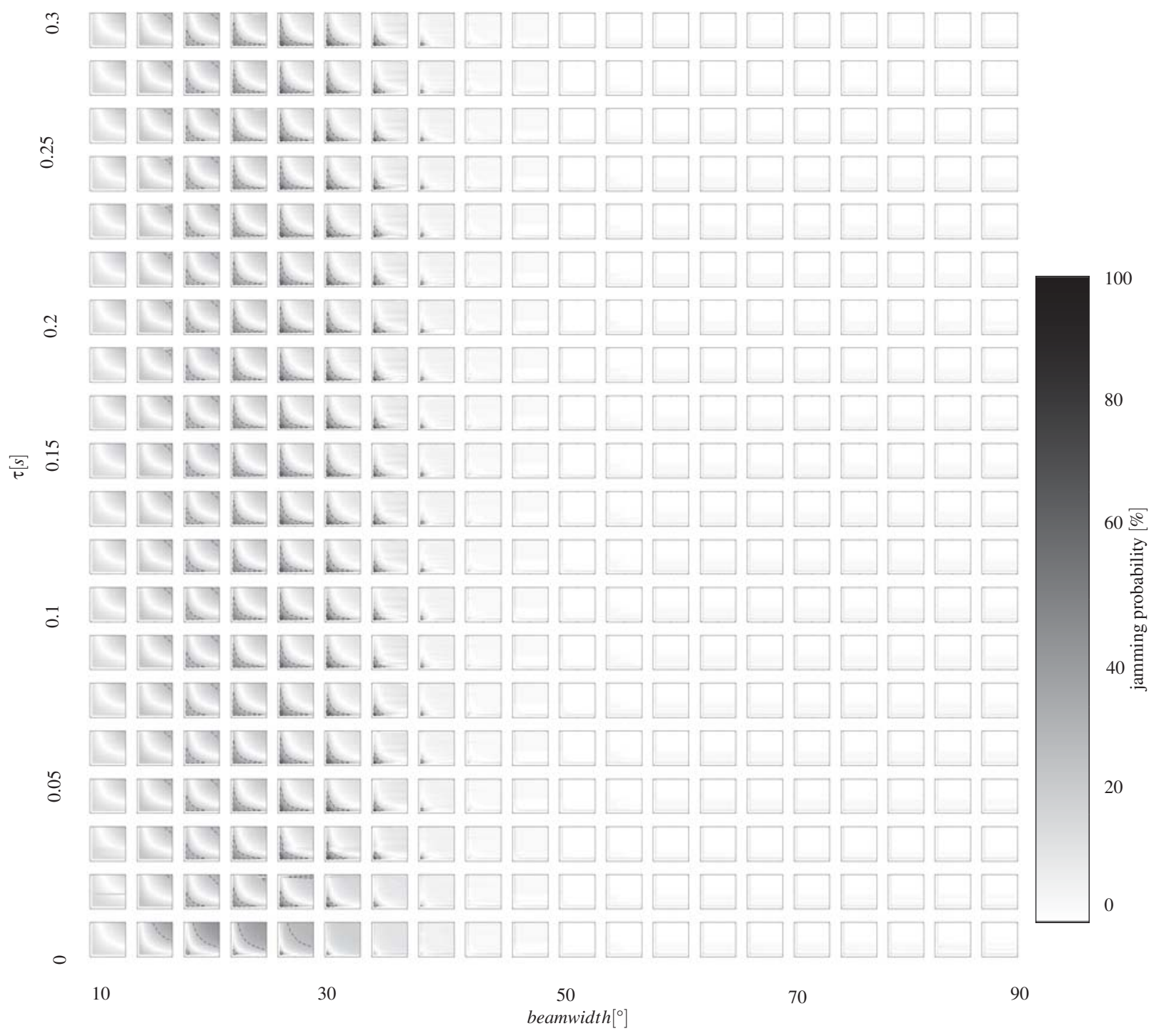

Figure 3.7: Fitting error - with four parameters: duty cycle, density, beamwidth, and time constant $\tau$

The result can also be shown as beamwidth and $\tau$ (which are two of the non-density 
parameters):

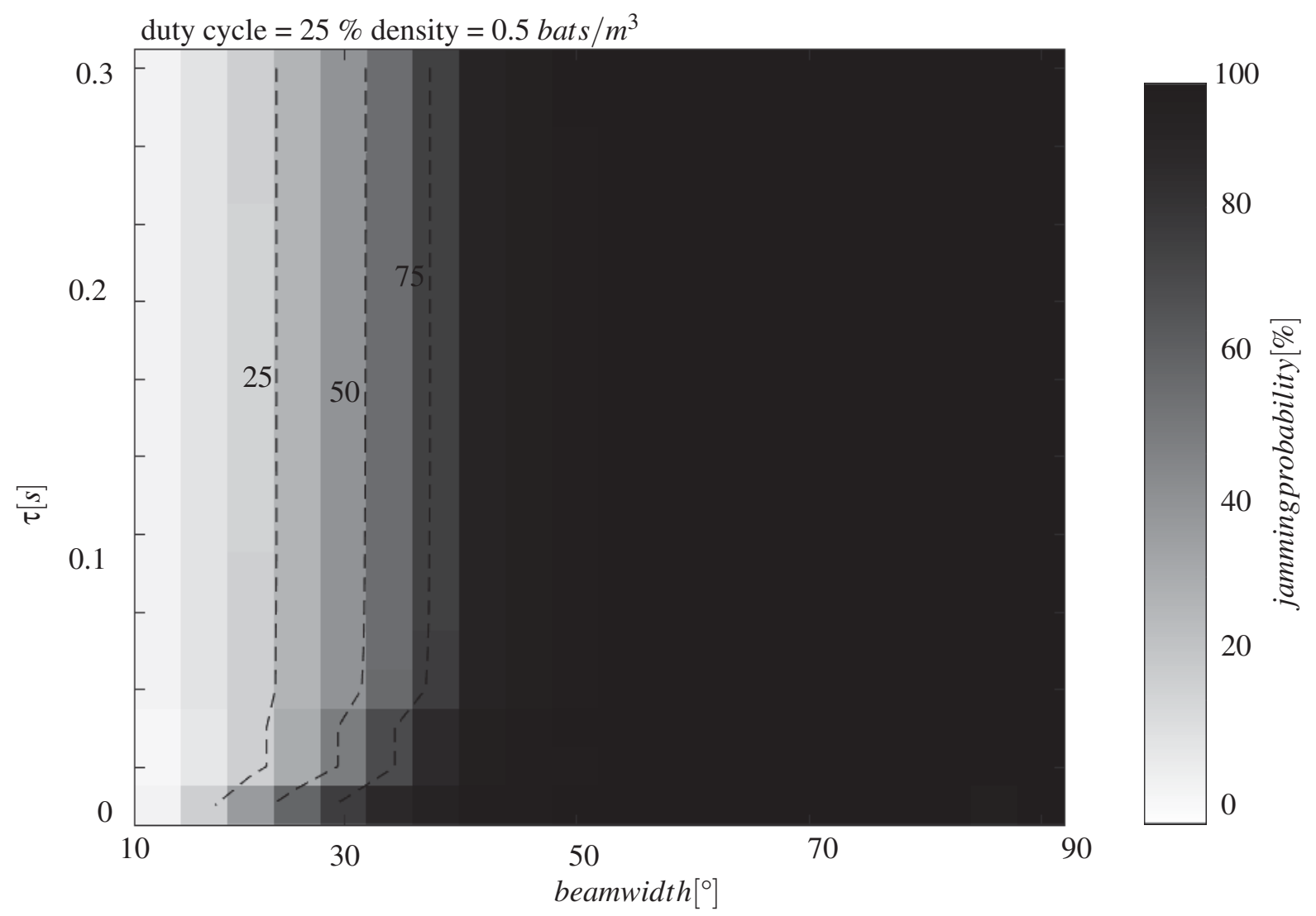

Figure 3.8: Result shown by jamming v.s. beamwidth \& $\tau$

Add the other two results, the overall simulation result is as following: 


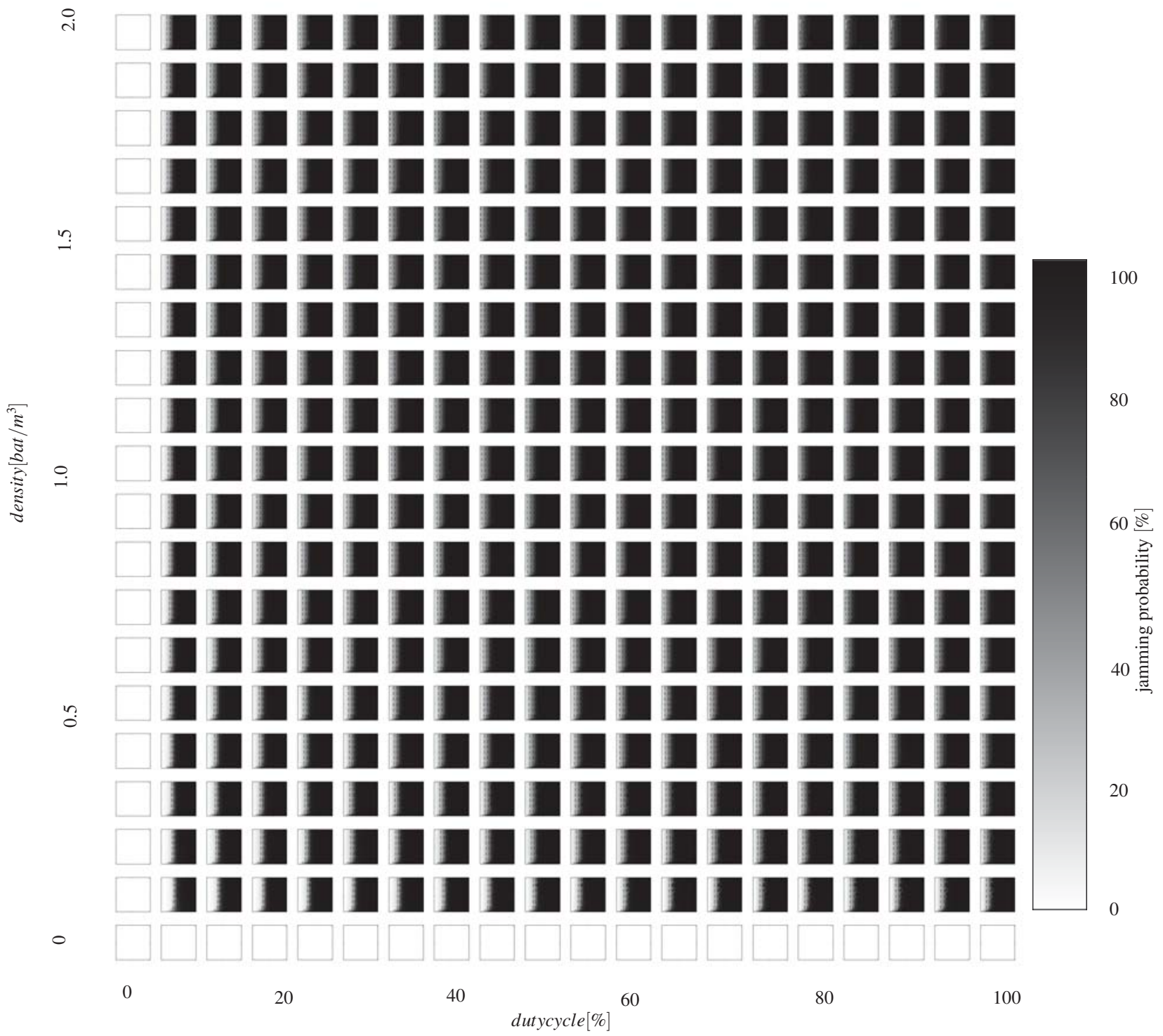

Figure 3.9: Simulation result v.s. four modeling parameters

The following is the fitting result: 


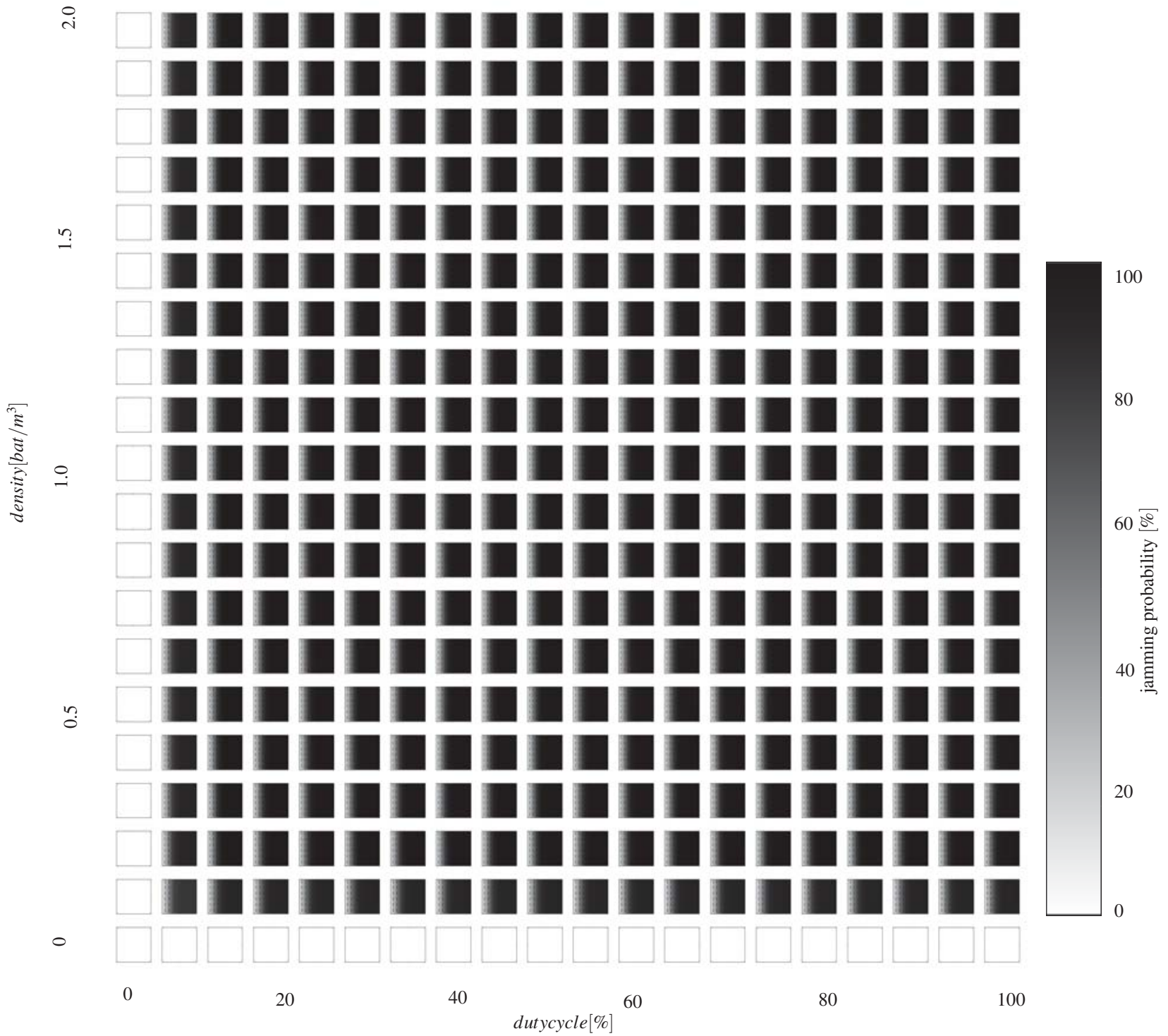

Figure 3.10: Fitting result

By comparing the simulation and fitting result, the fitting error can be obtained and shown as follow: 


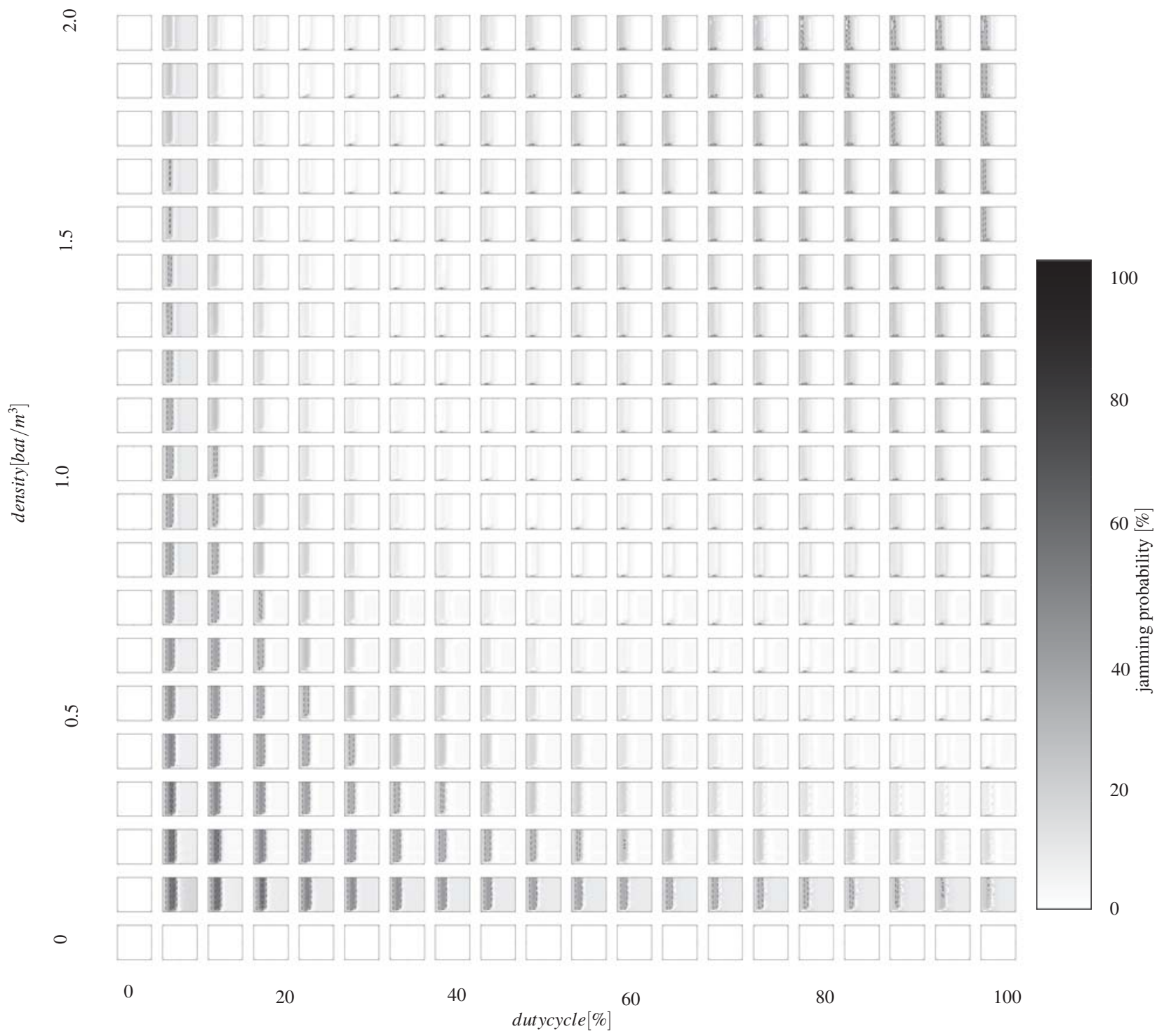

Figure 3.11: Absolute fitting error between simulation result and fitting result

In order to analysis the jamming contribution of jammed and not-jammed bat, we sort the contribution of input sound pressure amplitude from maximum to minimum and stair 
plot them. Comparison of the input sound amplitude and jamming threshold is plot and shown in Figure 3.12

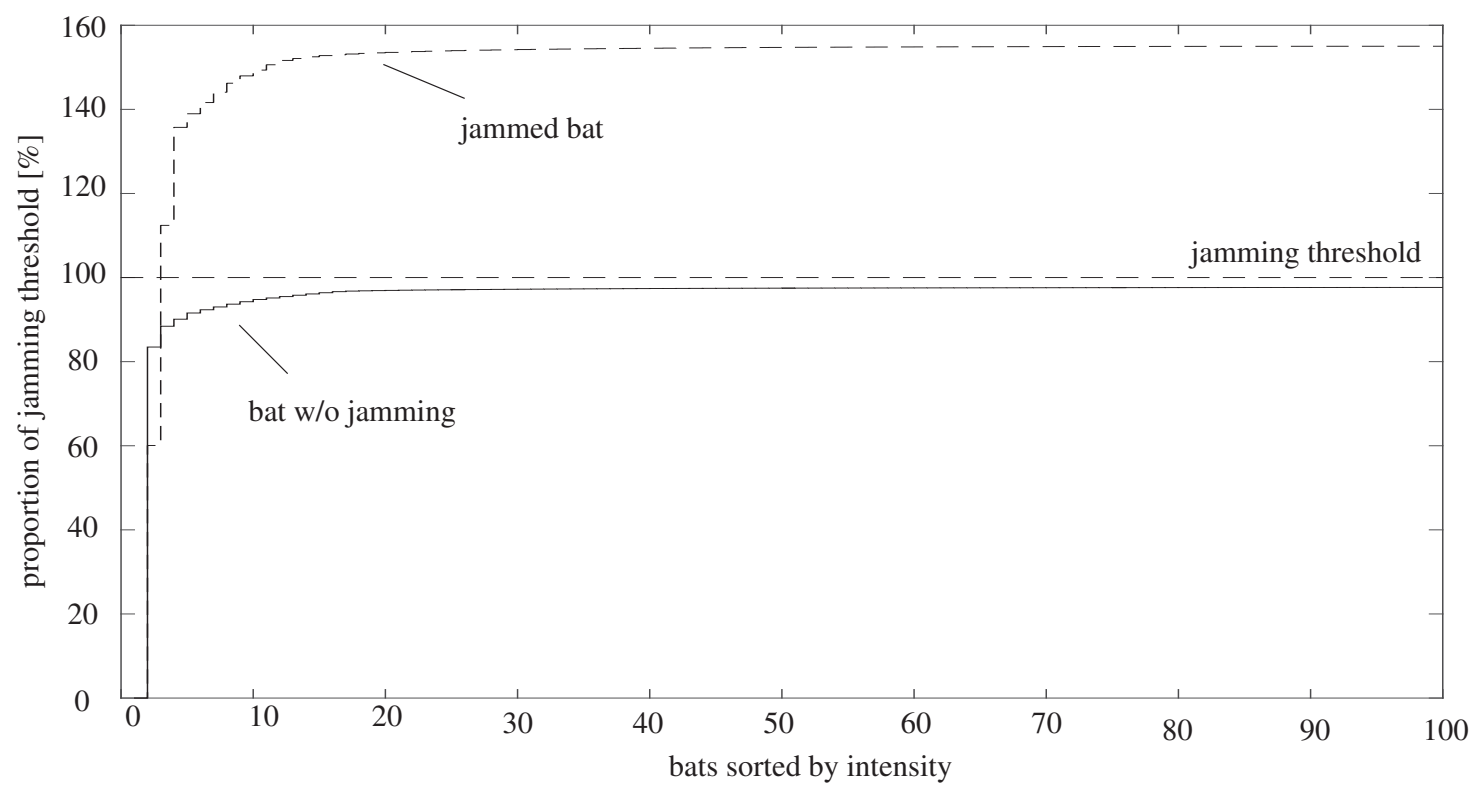

Figure 3.12: Example distributions of jamming input amplitudes for bat that is notjammed and jammed.(condition: time constant $\tau=0 \mathrm{~s}$, dutycycle $=100 \%$, beamwidth $=41^{\circ}$, density $=1$ bats $/ \mathrm{m}^{3}$ )

Spatial density and duty cycle are both density in space and time, which is two of the most important parameters. Here we plot all the combination of density and duty cycle, and try all possible value of beamwidth and time constant, to get the minimum and maximum as possible. The result is shown as follow: 

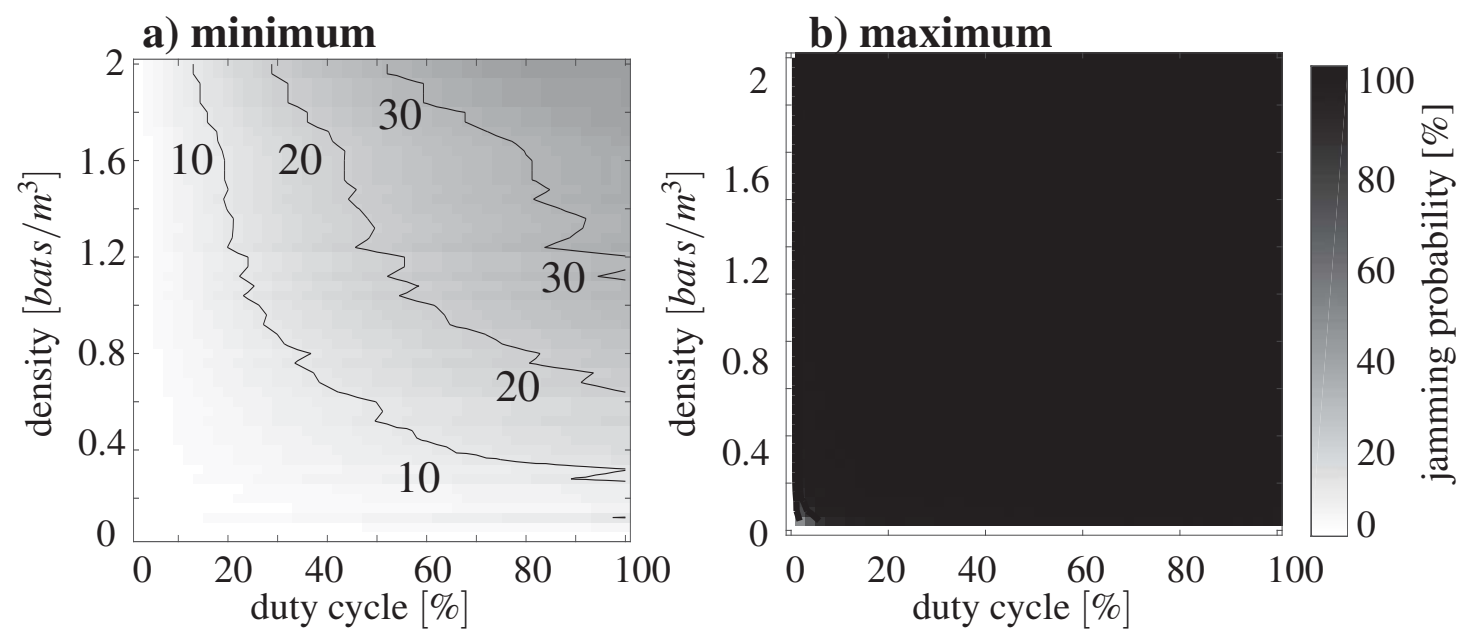

Figure 3.13: Jamming probability as a function of the model's two density parameters (bat spacial density and duty cycle) a) minimum jamming probability achievable, b) maximum jamming probability achievable by varying the beamwidth and the trajectory smoothness time constant $(\tau)$ over the ranges of $\left[10^{\circ}, 60^{\circ}\right]$ for beamwidth and $[0 \mathrm{~s}, 0.3 \mathrm{~s}]$ for the trajectory time constant. 


\section{Chapter 4}

\section{Discussion}

\subsection{Jamming contribution by individual parameters}

Among the four variables, the two parameters directly related to density in time and space were found to have the main impact on jamming probability, with the other two parameters contributing as well (Figure 3.1). Jamming probability increases either as density or duty cycle increases: jamming is zero when density or duty cycle is zero, and reaches the maximum point when duty cycle or density is high. Density and duty cycle play very similar role in contribution to jamming probability

Beamwidth has a very large impact on jamming probability around $30^{\circ}$, i.e. very low jamming when beamwidth is below $15^{\circ}$ and almost $100 \%$ jamming when beamwidth is above $35^{\circ}$. 
For medium values of beamwidth (between 15 and 40 degrees), the smoothness of the trajectory $(\tau)$ had an impact on jamming probability, but not for very small or larger beamwidth. As we can see from the analysis above that for beamwidth less than $15^{\circ}$ or more than $40^{\circ}$, jamming probability almost remain constant with varying time constant $\tau$. Only when beamwidth is between $[15,40]^{\circ}$ then time constant $\tau$ has some influence. In this region the jamming influence is also limited: the jamming probability will increase slightly when $\tau$ is very small, i.e., when the bats flying very wiggling trajectory. But other than that, the jamming probability remains almost the same across the entire range (as we can see from the figure above: jamming probability almost does not change for $\tau \sim[0.04,0.1])$.

From Figure 3.13 we can find out that for the same combination of duty cycle and density, jamming probability as low as 30\% and as high as $100 \%$ can be obtained by varying beamwidth and trajectory smoothness parameter value. This indicates that not all bat swarm have grave jamming problem, even with the same duty cycle and density parameters.

In summary, all four parameters have significant influence on the jamming probability. With the same configuration of other parameters, jamming and non-jamming situation can be achieved by varying one parameter.

\subsection{Jamming contribution by individual bat}

From Fig. 3.12 we can find out that the jammed bat's contribution from the most intensity one or two bats (out of 100 bats) is enough to cause the jammed bat to be jammed. That 
implies the jamming contribution is not evenly distributed among all inputs. In other words, if a bat is jammed, it is jammed by a few bats that have strong jamming contribution. This happens because a bat will seldom jam other bats, which depends on the right relative flying direction, relative distance and beamwidth. But when it jams, the jamming intensity will be very high.

\subsection{Fitting process matching simulation results}

As we can see from Fig. 3.7 and Fig. 3.11, the fitting error is small $(\leq 10 \%)$ for most of the regions. The fitting error is considered small and can represent the simulation result.

The fitting error for $\tau \sim[0,0.05] s$, beamwidth $\sim[30,50]^{\circ}$, is obvious because the fitting process can not capture the influence of $\tau$. $\tau$ 's influence on the jamming probability is only limited to certain combination of other parameter. For most of the combined set

of parameters $\tau$ have almost no effect. $\tau$ will have few effect except limited region (i.e. $\tau \sim[0,0.05] s$, beamwidth $\left.\sim[30,50]^{\circ}\right)$. The fitting process balance the fitting error by root mean square error and fit most of the region where $\tau$ has few effect. This phenomenon can be further explained by that actually those four parameters are not independent. The fitting error can be explained by the correlationship between beamwidth and $\tau$.

The fitting error for part of the region around $30^{\circ}$ beamwidth is due to the difference in transition slope between low and high beamwidth: the slope is smooth below $30^{\circ}$ but steep above $30^{\circ}$. The fitting process can not fit both at the same time, but equally evaluate 
both of them according to their weight difference (i.e. the difference in repetition time in the combination of four set of parameters). Since more simulation result comes with steep slope, then the fitting process follows this trend.

As shown in Fig. 3.7 and Fig. 3.11, the fitting error is very limited compared with the simulation result. This means the fitting result represents the simulation very well numerically. So with Eq. 3.3, a bat swarm researcher can estimate the probability of the jamming among the bat swarm by simply measure the four parameters: density $\rho$, trajectory smoothness $\tau$ (time constant), duty cycle $\epsilon$ and beamwidth $\sigma$. Since the four parameters are observable and quite easy to measure in lab or field, our result can be a simple and useful tool for field research scientist to assess the severity of jamming in a specific bat swarm.

\subsection{Limitation by finite simulation space}

The model assumes that the the space occupied by the bat swarm is infinite and the simulation just take a cubic space from the whole bat swarm. Ideally, to get the precise result, the simulation space should be infinite. But this can not achieved by the limited computer power. The simulation can only in limited space (i.e. $10 \times 10 \times 10=1000 \mathrm{~m}^{3}$ in this case) and there are constrains due to the limited space size. The finite simulation space can not represent the infinite model space. The difference in simulation space is obvious. In this simulation, multiple space size is examined and shown in the following figure: 


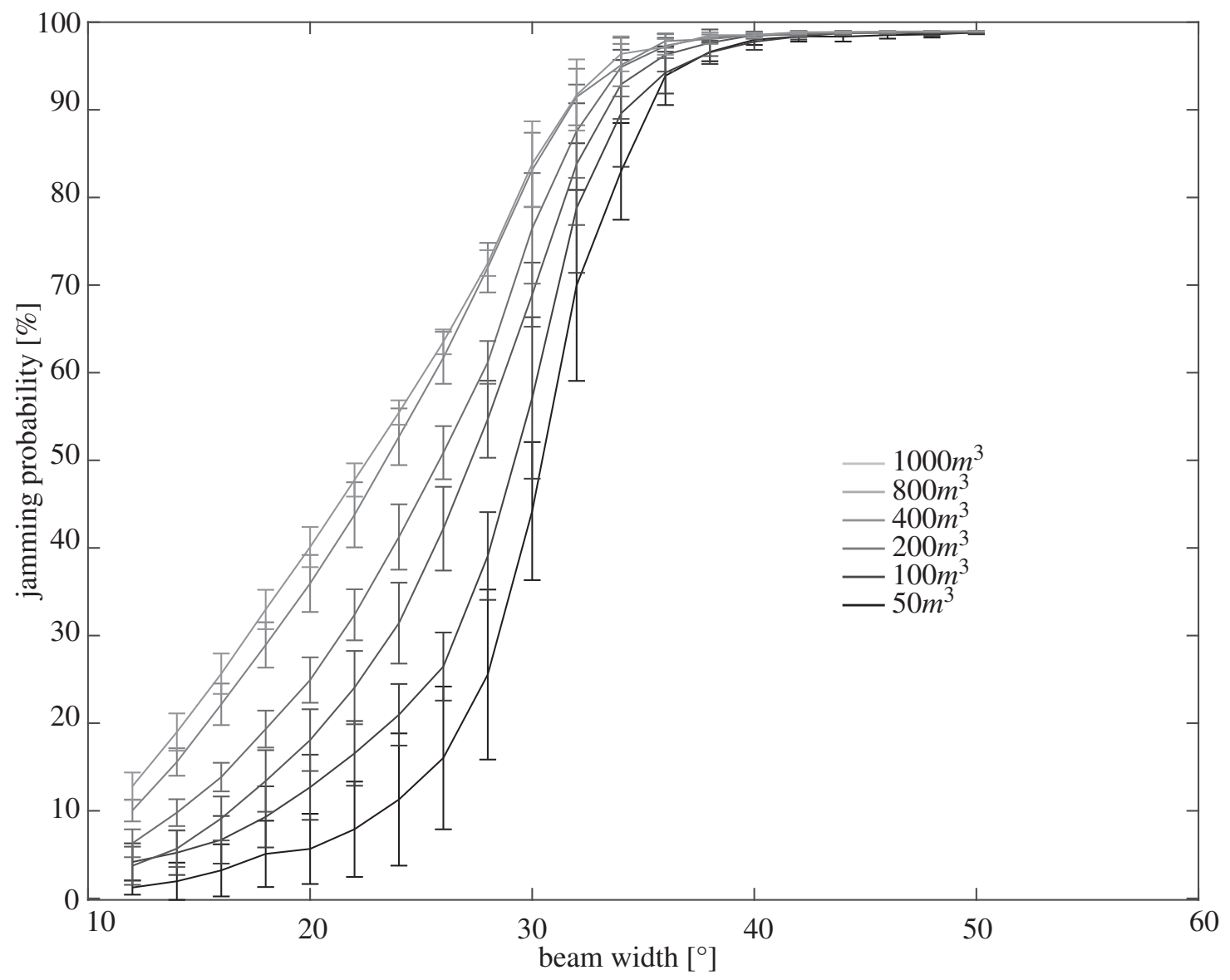

Figure 4.1: Influence of the simulation space on jamming probability, where duty cycle $=$ $100 \%$, and $\tau=0$ s.

As we can see from the figure above, the result difference due to difference in simulation space is large. However, the difference becomes small when the simulation space is beyond $800 \mathrm{~m}^{3}$. In this simulation, the simulation space is $1000 \mathrm{~m}^{3}$, which is with reasonable computer expenses. At the same time, the difference due to space difference reaches saturation there. 


\subsection{Further steps to improve the simulation}

In the simplified model, all bats are assumed to have identical frequency and their input to one bat is accumulated by considering their phase difference. However, different species of bats actually have different frequency (range). The same species will have different frequency individually, and even the same individual bat will have different frequency in different time. In order to come close to the real bat swarm, phase difference should be considered in the model.

The jamming threshold (which is defined as $\frac{1}{10}$ of the emission intensity $0.1 \mathrm{~m}$ right in front of the flying direction) is a estimation but doesn't have any field work or experiment to support. Next step, necessary field work should be done and test endurance of sonar input intensity level in different bat species. This is a step to help check the validity of the model.

The intensity field for both emission and reception are assumed to be identical, but actually bats flying in space will adjust their emission and reception direction. For future work, we might consider how bats make adjustment of this as well. Bats might also use the dynamics of bat's noseleaf and ear to avoid jammed by other bats.

In the model, all bats are assumed to distributed randomly in 3D space. However, in real caves, bats actually fly following a certain pattern. Instead of assuming all bats are randomly distributed, future work should be done to consider different flying patterns in bats. 


\section{Chapter 5}

\section{Future Work}

Based on the analysis result of this research, more work can be done to investigate and find out how to avoid jamming with each other. This can be applied to the new generation of air traffic control, allowing for more traffic flow manipulation, safer environment and better management.

Four parameters used here should be fairly easy to measure or estimate for actual bat swarms, the model predictions could be used to decide quickly if bats in certain situation are likely to suffer from substantial jamming probabilities. This research could guide researchers investing possible mechanisms for jamming avoidance to scenarios where such mechanisms are the most likely to be in operation.

As the increase of air traffic flow, especially as the soaring of drones due to its wide range of applications such as deliver objects, rescue, and aerial photographs. With all kinds 
of air vehicles in the dense air, there comes the problem of traffic control. The demand to manage air traffic efficiently and safely is increasing. Current air traffic control mostly rely on traffic tower and radar, all flights are under tight control, i.e. you have to apply to fly in certain spot and certain time and have to fly in that specific spot and time in order avoid crash into each other. The centralized control is safe but the traffic flow is limited. As the demand increases, this centralized control method can not meet the demand any more.

However, when we take a close look at the bat swarm, they do not have any central coordination, i.e. they do not rely on centralized control, but de-centralized control. They can fly in any time, any location, any direction, just as they want. Yet we are amazed to find out that rare occasions will they collide into each other, even in caves with tens of thousands of bats flying in and out at the same time in a small opening. This paper is the first step to look into how bats interact with each other. New air traffic control methods might be found inspired by compare the similarity and differences of bat swarm and air traffic flow. 


\section{Chapter 6}

\section{Conclusion}

\subsection{A simple model for bat swarm}

This research came up with a simple numerical model for bat swarm, with only four parameters: density, duty cycle, beamwidth, and trajectory smoothness time constant $\tau$. Simulation is done based on this simplified bat swarm model, by having bats flying in a $10 m \times 10 m \times 10 m=1000 m^{3}$ space and interact with each other acoustically. Analysis is done based on the simulation result and conclusion is made from the analysis. With these four parameters, the simplified bat swarm model captures the main characteristics of the real bat swarm. 


\subsection{All four parameters are critical to jamming situa- tion}

Density and duty cycle, which spatial density and temporal density, are two of most important parameters. However, non jamming and serious jamming situation can be created even with the same set of density and duty cycle configuration, by varying beamwidth and $\tau$ values. This indicates that non-density parameters, i.e. beamwidth and $\tau$ can also have large impact on jamming probability. The intuitive understanding of this is that not only the density and duration calls of the bats will influence the jamming situation, but also how the bats behaves: what kind of trajectory they fly and what kind of beam shape they have. This indicates all parameters are important for the model.

\subsection{Not every bat swarm experience jamming}

In the model, jammed and not jammed situation can be achieved by tuning any one of the swarm parameters: density, duty cycle, beamwidth, and trajectory smoothness. With the same configuration of a set of two parameters, serious or no jamming situation can be achieved by varying the other two parameters. With the right configuration of trajectory smoothness and beamwidth, many jamming scenarios can be avoided. This indicates not every bat swarm necessary experience jamming, even with the same situation, bats can change the call duration, beampattern shape, and the trajectory they fly to avoid jamming. 


\section{Bibliography}

[1] J. K. Parrish, W. M. Hamner, "Animal Groups in Three Dimensions," Cambridge University Press (1997).

[2] M. W. Moffett, "Sociobiology of the Ant of the Genus Pheidologeton," Dissertation, Harvard University (1987).

[3] T. J. Pitcher, B. L. Partridge, "Fish School Density and Volume," Mar. Biol. 54, 383394 (1979).

[4] M. Ballerini, "Interaction ruling animal collective behavior depends on topological rather than metric distance: Evidence from a field study," PNAS. 105, 1232-1237 (2008).

[5] L. D. Mech, L. Boitani, "Wolves: behavior, ecology, and conservation," The University of Chicago Press (2006).

[6] M. B. Fenton and J. M. Ratcliffe, "Bats," Curr. Biol. 20, 1060-1062 (2010). 
[7] M. E. Bates, S. A. Stamper and J. A. Simmons, "Jamming avoidance response of big brown bats in target detection," J. Exp. Biol. 211, 106-113 (2008).

[8] E. H. Gillam, N. Ulanovsky, and G. F. McCracken, "Rapid jamming avoidance in biosonar," Proc. R. Soc. B 274, 651-660 (2007).

[9] E. H. Gillam, B. K. Montero, "Influence of call structure on the jamming avoidance response of echolocating bats," J. Mammal. 97, 14-22 (2015).

[10] N. Ulanovsky, M. Brock Fenton, A. Tsoar and C. Korine, "Dynamics of jamming avoidance in echolocating bats," Proc. R. Soc. Lond. B. 271, 1467-1475 (2004).

[11] C. Ibanez, J. Juste, R. Lopez-Wilchis, and A. Nunez-Garduno "Habitat variation and jamming avoidance in echolocation calls of the sac-winged bat (Balantiopteryx Plicata)," J. Mammal. 85, 38-42 (2004).

[12] C. Chiu, W. Xian, and C. F. Moss. "Flying in silence: Echolocating bats cease vocalizing to avoid sonar jamming," PNAS. 105, 13116-13121 (2008).

[13] J. Jarvis, W. Jackson and M. Smotherman, "Groups of bats improve sonar efficiency through mutual suppression of pulse emissionss," Front. Phys. 4, article 140. (2013).

[14] J. Jarvis, K. M. Bohn, J. Tressler, M. Smotherman, "A mechanism for antiphonal echolocation by free-tailed bats," Anim. Behav. 79, 787-796. (2010).

[15] E. Amichai,G. Blumrosen, and Y. Yovel, "Calling louder and longer: how bats use 
biosonar under severe acoustic interference from other bats" Proc. R. Soc. B 282, 20152064. (2015).

[16] J. M. Ratcliffe, H. M. ter Hofstede, R. Avila-Flores, M. B. Fenton, G. F. McCracken, S. Biscardi, J. Blasko, E. Gillam,J. Orprecio, and G. Spanjer, "Conspecifics influence call design in the Brazilian free-tailed bat, Tadarida brasiliensis" Can. J. Zool. 82, 966-971. (2004).

[17] V. Necknig, A. Zahn, "Between-species jamming avoidance in Pipistrelles?" J. Comp. Physiol. A. 197, 469-473. (2011).

[18] J. P. Balcombe, and M. B. Fento, "Eavesdropping by Bats: The Influence of Echolocation Call Design and Foraging Strategy" Ethology 79, 158-166. (1988).

[19] N. Cvikel, E. Levin, E. Hurme, I. Borissov, A. Boonman, E. Amichai, Y. Yovel, "Onboard recordings reveal no jamming avoidance in wild bats," Proc. R. Soc. B 282, 20142274. (2015).

[20] M. B. Fenton, P. A. Faure and J. M. Ratcliffe, "Evolution of high duty cycle echolocation in bats," J. Exp. Biol. 215, 2935-2944 (2012).

[21] N. I. Hristov, M. Betke, E. H. Diane, Theriault, Angshuman bagchi, and Thomas H. Kunz, "Seasonal variation in colony size of Brazilian free-tailed bats at Carlsbad Cavern based on thermal imaging," J. Mammal. 91, 183-192, (2010). 
[22] L. Jakobsen, J. M. Ratcliffe and Annemarie Surlykke "Convergent acoustic field of view in echolocating bats," Nature. 493, 93-96 (2013).

[23] L. Jakobsen and A. Surlykke, "Vespertilionid bats control the width of their biosonar sound beam dynamically during prey pursuits," PNAS. 107, 13930-13935 (2010).

[24] N. Matsuta, S. Hiryu, E. Fujioka, Y. Yamada, H. Riquimaroux, Y. Watanabe, "Adaptive beam-width control of echolocation sounds by CFFM bats, Rhinolophus ferrumequinum nippon, during prey-capture flight," J. Exp. Biol. 216, 1210-1218 (2013).

[25] P. Kounitsky, J. Rydell, E. Amichai, A. Boonman, O. Eitan, A. J. Weiss, Y. Yovel, "Bats adjust their mouth gape to zoom their biosonar field of view" PNAS. 112, 6724-6729 (2015).

[26] B. Hayward and R. Davis "Flight Speeds in Western Bats," J. Mammal. 45-2, 236-242 (1964).

[27] P. A. Martin and R. A. Dalrymple, "Scattering of long waves by cylindrical obstacles and gratings using matched asymptotic expansions," J. Fluid Mech. 188, 465-490 (1988).

[28] K. Ghose and C. F. Moss, "The sonar beam pattern of a flying bat as it tracks tethered insects," J. Acoust. Soc. Am. 114, 1120-1131 (2003).

[29] A. Surlykke, S. B. Pedersen and L. Jakobsen, "Echolocating bats emit a highly directional sonar sound beam in the field," Proc. R. Soc. B. 276, 853-860 (2009). 
[30] L. Jakobsen, M. N. Olsen, and A. Surlykke, "Dynamics of the echolocation beam during prey pursuit in aerial hawking bats," PNAS. 112, 8118-8123 (2015).

[31] L. Jakobsen, A. Surlykke, "Vespertilionid bats control the width of their biosonar sound beam dynamically during prey pursuit," PNAS. 107, 13930-13935 (2010). 


\section{Appendix A}

\section{Program Source}

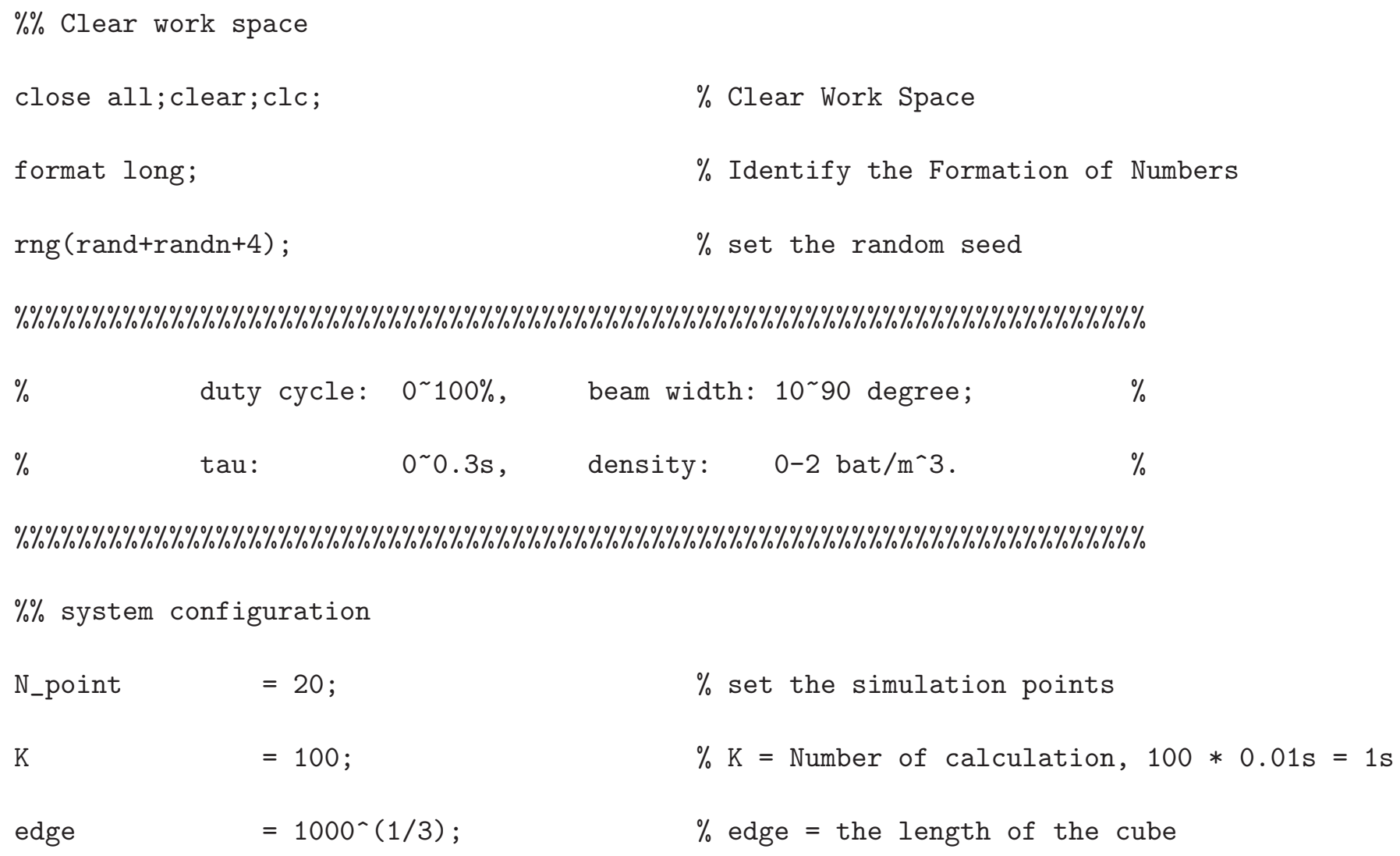


$d t$

tau_all

alpha_all

duty_all

beam_all

density_all

jamming_ave

$=\operatorname{zeros}\left(1, N_{-}\right.$point $)$;

posotion_speed_x $=5 * d t: 5 * d t: 5 * d t * K$;

$\%$ running

for iii $=1: N_{-}$point

tic;

$N=$ density_all(1,iii);

speed $=\operatorname{zeros}(3, N, K)$;

$\%$ get initial position

position_original $=(\operatorname{randn}(3, N) / 6+0.5) *$ edge;

position_original $(1,:)=$ position_original $(1,:) / 10$;

noise $=10 * d t *(\operatorname{rand}(3, N, K)-0.5)$;

for $j j j=1:$ N_point

position $=\operatorname{zeros}(3, N, K)$;

alpha $=$ alpha_all $(1, j j j)$

for $n=1: N$

$\%$ smooth trajectory

for $k=2: k$
$\%$ smoothness

$$
\text { position }(:, \mathrm{n}, \mathrm{k})=(1-\mathrm{alpha}) * \operatorname{noise}(:, \mathrm{n}, \mathrm{k})+\operatorname{alpha} * \operatorname{position}(:, \mathrm{n}, \mathrm{k}-1)
$$


end

end

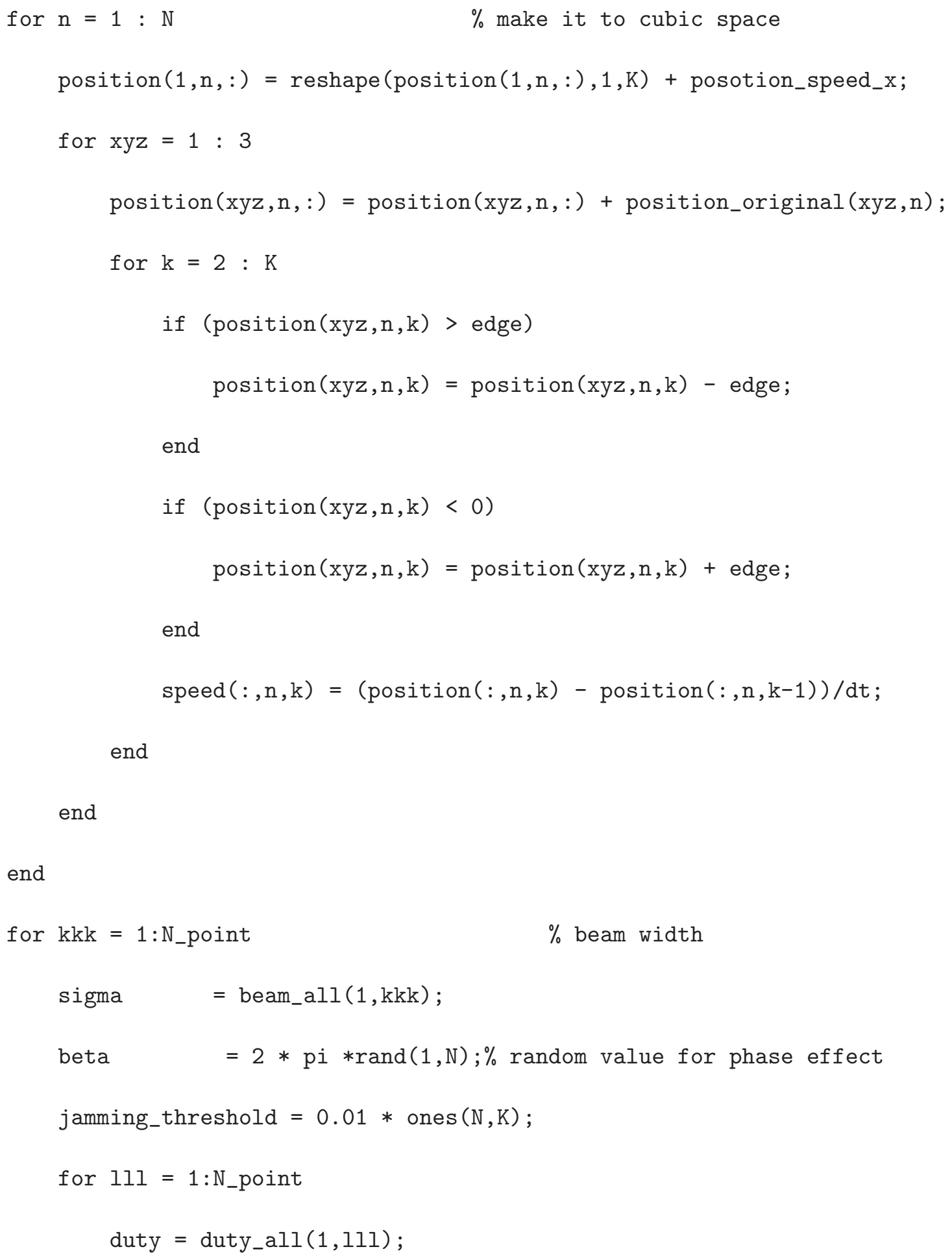




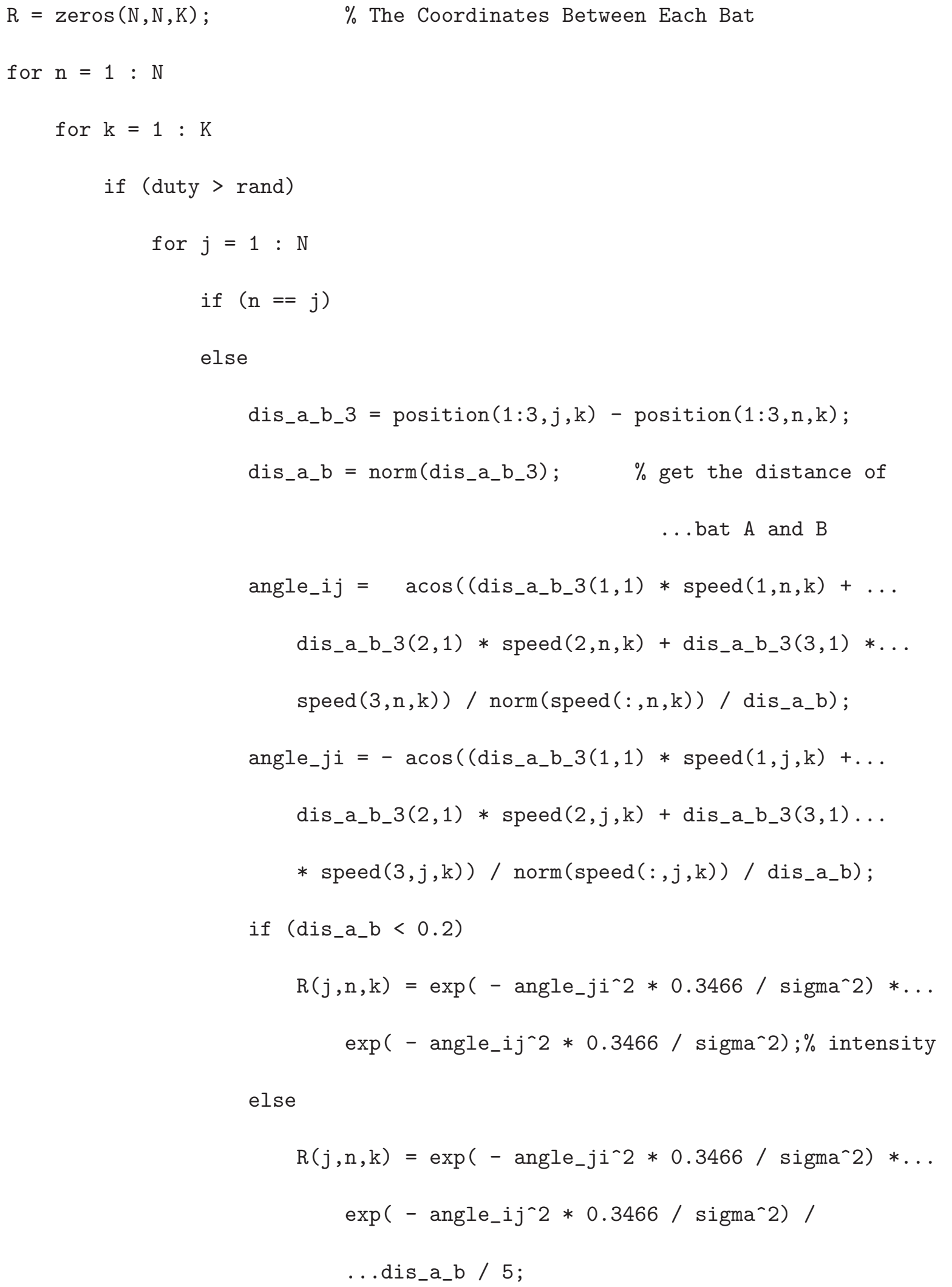


end

end

end

end

end

end

alpha $\quad=0 ; \quad \%$ add $\sin$

noise_sum_sin $\quad=\operatorname{zeros}(\mathrm{N}, \mathrm{K})$;

for $k=1: K$

for $n=1: N$

for $j=1: N$

$A=$ noise $\_$sum_sin $(n, k) ;$

$B=R(n, j, k)$;

if $i \operatorname{snan}(B)|| B==0$

else

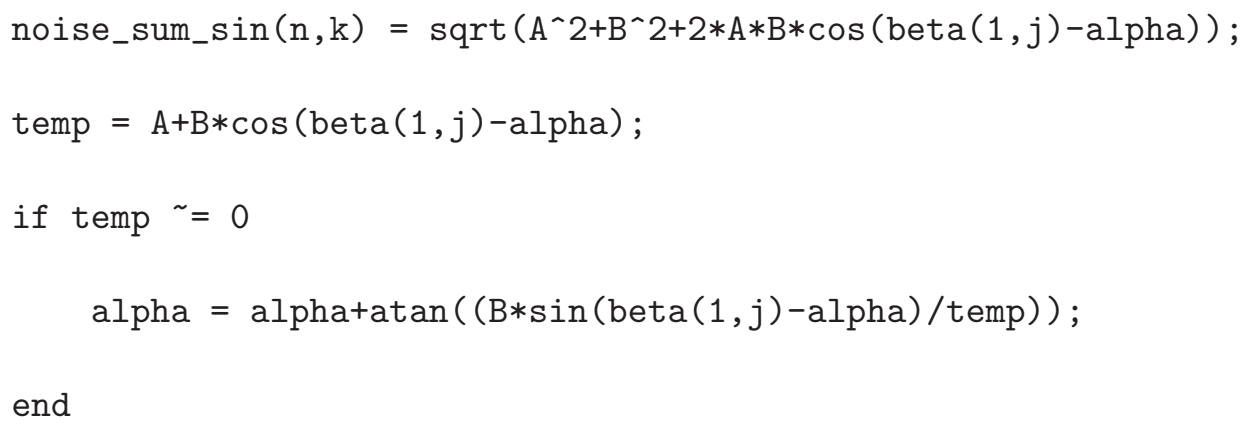


end

jamming $=$ noise_sum_sin $>$ jamming_threshold;

jamming_ave $(1,111)=\operatorname{sum}(\operatorname{sum}($ jamming $)) / \mathrm{N} / \mathrm{K} * 100$;

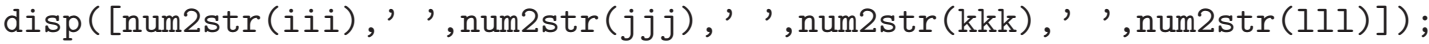

$\operatorname{disp}\left(j a m m i n g \_a v e(1,111)\right)$;

number $=\operatorname{strcat}($ num2str $(i i i), \operatorname{num} 2 \operatorname{str}(j j j)$, num2str $(k k k)) ;$

save(['jamming', number, '.mat'] , 'jamming_ave');

end

number $=\operatorname{strcat}($ num2str $(i i i)$, num2str $(j j j)$, num2str $(k k k)) ;$

save(['jamming', number, '.mat'] , 'jamming_ave');

toc;

end

end

end 